\title{
Building blocks of closed and open string amplitudes
}

\author{
Pierre Vanhove $^{a, b, *}$ and Federico Zerbini ${ }^{c}$ \\ ${ }^{a}$ Institut de Physique Théorique \\ CEA, IPHT, F-91191 Gif-sur-Yvette, France \\ CNRS, URA 2306, F-91191 Gif-sur-Yvette, France \\ ${ }^{b}$ National Research University Higher School of Economics, Russian Federation \\ ${ }^{c}$ Institut de Recherche Mathématique Avancée (IRMA) \\ Université de Strasbourg \\ 7 rue René Descartes, 67084 Strasbourg, France
}

\begin{abstract}
In this text we review various relations between building blocks of closed and open string amplitudes at tree-level and genus one. We explain that KLT relations between tree-level closed and open string amplitudes follow from the holomorphic factorisation of conformal correlation functions on conformal blocks. We give a simple hands-on evaluation of the $\alpha^{\prime}$-expansion of tree-level closed string amplitudes displaying the special single-valued nature of the coefficients. We show that the same techniques can be used also at genus-one, where we give a new proof of the single-valued nature of the coefficients of 2-point closed string amplitudes. We conclude by giving an overview of some open problems.
\end{abstract}

MathemAmplitudes 2019: Intersection Theory \& Feynman Integrals (MA2019)

18-20 December 2019

Padova, Italy

*Speaker 


\section{Part I}

\section{Introduction}

We are still uncovering remarkable properties of string theory amplitudes. Perturbative string theory is characterised by a series expansion with respect to the string coupling constant $g_{s}$. At each order in perturbation theory one can consider the expansion in the small inverse string tension $\alpha^{\prime}$ leading to quantum field theory scattering together with an infinite set of stringy quantum corrections. This double expansion can reveal a lot of deep properties of the low-energy effective action of quantum gravity on various background configurations.

It is remarkable that the ultra-violet divergences of maximal supergravity amplitudes in various dimensions [1,2] are in perfect agreement with the low-energy expansion of string theory amplitudes up to genus three and are fully compatible with the non-perturbative U-duality symmetries of string theory [3-13]. Recent works have shown a perfect match between this double expansion and the flatspace limit of correlation functions in $\operatorname{AdS}_{5} \times S^{5}$, giving a remarkable confirmation of the AdS/CFT correspondence [14-17].

There are five consistent supersymmetric string theories in ten dimensions composed of four closed superstring theories-the type IIA, type IIB and the two heterotic strings-and the type I superstring theory, which contains also open strings [18-20]. These string theories are unified by duality symmetries [21, 22] which are the landmark of the special mathematical and physical properties of string theory. In this text we are interested in the perturbative expansions of closed and open string amplitudes, and how they are related.

The type I superstring theory is an orientifold of the closed string type IIB theory obtained by quotienting by the action of the world-sheet parity reversal $\Omega:(\sigma, \tau) \rightarrow(2 \pi-\sigma, \tau)$ (see [23] for a general review of orientifolds). At each order in perturbation, the type I superstring amplitude involves a sum of two-dimensional super-surfaces obtained from the action of the involution $\mathcal{I}(z)$, induced by the orientifold action $\Omega$. The type I superstring theory contains open and closed strings, possibly non-oriented, which lead to anomaly cancellation [24, 25] and Ramond-Ramond tadpole cancellation [23, 26].

Closed (oriented) string amplitudes, which appear in the four closed superstring theories, are computed by integrals over the moduli space of super-Riemann surfaces of genus $h$, the number of handles, with $n$ punctures and no boundaries. Each order in perturbation theory is weighted by the string coupling constant $g_{s} \ll 1$ to the power of the Euler characteristic of the surface, in this case $g_{s}^{2 h-2}$.

In type I superstring theory, involutions $\mathcal{I}(z)$ act on the (closed) super-Riemann surfaces to remove $b$ discs and add $c$ cross-caps (obtained by gluing a real projective plane) to the surface. The loci of fixed points of the involution are the boundaries of the world-sheet, which contain the open string insertions. Each order in perturbation is weighted by the string coupling constant $g_{s} \ll 1$ to the power of the Euler characteristic of the surface, in this case $g_{s}^{2 h+b+c-2}$.

On a surface $M$ obtained by applying an involution $\mathcal{I}_{M}(z)$ to a closed (oriented) world-sheet, the 
Green function is given in terms of the closed string Green function by the formula [27, 28]:

$$
G^{M}\left(z, z^{\prime}\right)=\frac{1}{2}\left(G^{\text {closed }}\left(z, z^{\prime}\right)+G^{\text {closed }}\left(\mathcal{I}_{M}(z), z^{\prime}\right)+G^{\text {closed }}\left(z, \mathcal{I}_{M}\left(z^{\prime}\right)\right)+G^{\text {closed }}\left(\mathcal{I}_{M}(z), \mathcal{I}_{M}\left(z^{\prime}\right)\right)\right)
$$

At tree-level, the Kawai, Lewellen and Tye (KLT) relations [29] give a construction of the closed string integrals on the sphere with $n$ punctures as a sum of products of pairs of open string amplitudes with $n$ marked points on the boundary of the disc. This relation has led to the fundamental perturbative duality between colour factors of gauge theory and kinematic factors in field theory and string theory [30, 31].

Hints that similar relations may generalise at genus one come from the small $\alpha^{\prime}$ expansion of one-loop open and closed string amplitudes [32-37]. Much less is known at higher-loop orders but one can reasonably suspect that similar relations exist as being a consequence of the intrinsic relation between closed and open string amplitudes.

With the aim of finding a higher-loop generalisation of the KLT relations in string theory, we have shown in [38] that the relation between closed string tree-level amplitudes and open string tree-level amplitudes is a particular application of the conformal block decomposition of correlation functions in two-dimensional minimal model conformal field theory. This construction is generalizable at higher genus [39, 40]. The chiral splitting of the closed string conformal blocks at fixed internal momenta gives a realisation of the double-copy of open string integrands before integration over the loop momentum (see [41] for a discussion at genus one). Therefore, this approach may lead to higher-loop generalisation of the KLT relations in string theory.

The conformal block approach, and its restriction to amplitudes given by the KLT relation, inverts the involution construction for tree-level amplitudes, as illustrated on the following diagram:

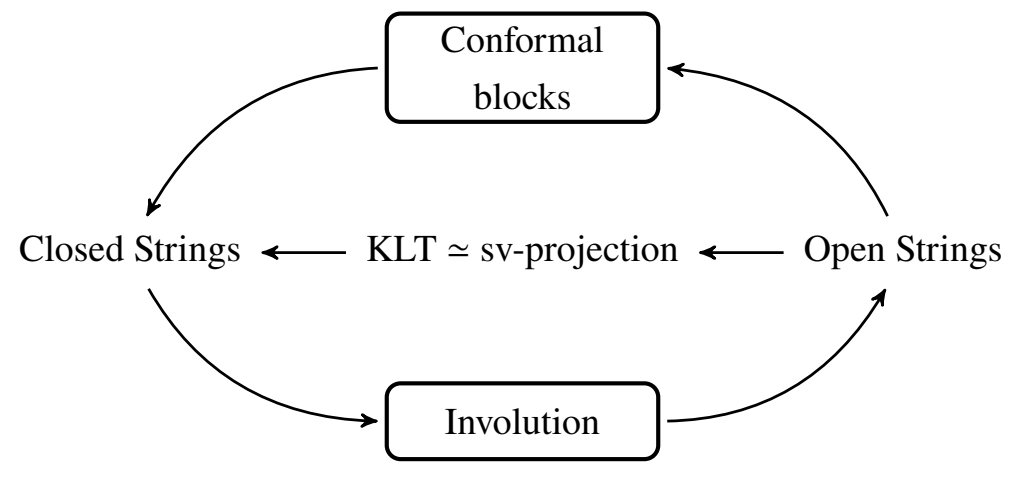

This article is divided into three parts, and the introduction constitutes the first one. Part II is about the building blocks of tree-level perturbative string amplitudes. We start by reviewing the result [42-48] that all tree-level (bosonic or type I super-) string amplitudes on the disc (with both open and closed string states) and on the real projective plane (where only closed string states appear) are combinations of open string building blocks given by ordered integrals on the boundary of the disc. In a similar way, tree-level closed (bosonic, type IIA or type IIB or heterotic) string amplitudes are combinations of closed string building blocks. All tree-level (bosonic or super-) string amplitudes 
are obtained from appropriate choices of the kinematic coefficients in the linear combinations of the building blocks.

We then give the relation between the open string and closed string building blocks using the conformal block decomposition approach of [38]. The open string building blocks are special values of the (multi-valued) conformal blocks and the closed string building blocks are special values of (single-valued) conformal correlation functions. The modern formulation of the KLT relations in terms of twisted cohomology theory as twisted bilinear period relations $[49,50]$ should generalise and give in this context a cohomological interpretation of the conformal block decomposition.

The cohomological viewpoint can be used to relate closed string amplitudes to the newborn theory of single-valued periods [51,52], providing a mathematical interpretation of the fact that open and closed string amplitude building blocks are special values of multi-valued and single-valued functions, respectively. This explains why the $\alpha^{\prime}$-expansion of the closed string building blocks involves only a very small subspace of multiple zeta values, given precisely by those called "singlevalued" which come from the theory of single-valued periods [38, 50, 53-57]. Following [38], we illustrate this phenomenon by giving a simple hands-on evaluation of the $\alpha^{\prime}$-expansion of closed string amplitudes based on the "single-valued integration" of (single-valued) multiple polylogarithms developed in $[38,58,59]$.

Part III is about the building blocks of one-loop perturbative string amplitudes. On the one hand, we have closed (oriented) superstring amplitudes on the torus, arising from the four closed superstring theories. They can be expanded on closed string building blocks as in the genus zero case. The lowenergy expansion of these building blocks gives rise to modular graph functions and forms [33, 60-62], which will be reviewed in section 4.1 and which are conjecturally related to the same single-valued periods appearing at genus zero. In particular, in section 4.2 we present a new proof of a special case of this conjecture, adapting to this setting the single-valued integration method already used at tree level. Even though this is not a new result (two different proofs have already appeared in the recent papers [36, 63]), our new approach may be of interest because it seems more likely to generalize.

On the other hand, we have type I superstring amplitudes given by applying involutions on the torus. One finds superstring amplitudes on the annulus (with both open and closed string states), on the Möbius strip (unoriented, with both open and closed string states) and on the Klein bottle (unoriented, only closed string states appear). The low-energy expansion of amplitudes with open string states on the boundaries of the annulus or the Möbius strip involves elliptic analogues of multiple zeta values [64-66]. In this case, it was observed in [32] that open and closed string building blocks seem to be related by a genus-one analogue of the single-valued projection from genus-zero. This points towards the possibility of extending KLT relations to genus one. In section 5.1 we briefly review the simplest instance of this relation, which was demonstrated in [36].

Finally, very little is known about the low-energy expansion of one-loop string amplitudes with both open and closed string states. For instance, we do not know if it is possible to reduce them to open string building blocks (annulus amplitudes with boundary insertions only), as in the tree-level case. This is one of the open questions that we list in section 5.2, which concludes the paper. 


\section{Acknowledgments}

We would like to thank C. Angelantonj, O. Schlotterer, P. Tourkine for discussions and comments on this text. P. Vanhove thanks the organisers of the workshop "MathemAmplitudes 2019: Intersection Theory and Feynman Integrals" held in Padova, Italy on 18-20 December 2019, for giving the opportunity to present some of the results reported in this text. The research of P. Vanhove has received funding from the ANR grant "New Structures in Amplitudes" ANR-17- CE31-0001-01, and is partially supported by Laboratory of Mirror Symmetry NRU HSE, RF Government grant, ag. $\mathrm{N}^{\circ}$ 14.641.31.0001. The research of F. Zerbini was supported by the LabEx IRMIA.

\section{Part II}

\section{Tree-level amplitudes}

\section{Building blocks of closed and open string amplitudes}

\subsection{Closed oriented tree-level amplitudes}

Any tree-level $N+3$-point closed oriented string amplitude $(N \geq 1)$ can be written as a finite linear combination of partial amplitudes [18, 19, 67]

$$
M_{N+3}(\boldsymbol{s}, \boldsymbol{\epsilon})=\sum_{r} c_{r}(\boldsymbol{s}, \boldsymbol{\epsilon}) M_{N+3}\left(\boldsymbol{s}, \boldsymbol{n}^{r}, \tilde{\boldsymbol{n}}^{r}\right) .
$$

The coefficients $c_{r}(\boldsymbol{s}, \boldsymbol{\epsilon})$ are rational functions of the kinematic variables $\boldsymbol{s}=\left(s_{i j}\right)_{1 \leq i<j \leq N+3}:=\left(-\frac{1}{2} k_{i}\right.$. $\left.k_{j}\right)_{1 \leq i<j \leq N+3}$ (where $k_{i}$ are external momenta, subject to momentum conservation $k_{1}+\cdots+k_{N+3}=0$ and on-shell condition $\alpha^{\prime} k_{i}^{2} \in 4 \mathbb{Z}$ lower or equal to 4 with the metric convention $\left.(-,+, \ldots,+)\right)$, the polarisation tensors $\boldsymbol{\epsilon}=\left(\epsilon_{i}\right)_{1 \leq i \leq N+3}$ and the colour factors for the heterotic string amplitudes. These kinematic coefficients determine the specifics of the closed string theory (bosonic string, superstring or heterotic string) or of the external states (polarisation tensors and colour factors). The partial amplitudes $M_{N}(\boldsymbol{s}, \boldsymbol{n}, \tilde{\boldsymbol{n}})$, defined for integers $\boldsymbol{n}=\left(n_{i j}\right)_{1 \leq i<j \leq N+3}, \tilde{\boldsymbol{n}}=\left(\tilde{n}_{i j}\right)_{1 \leq i<j \leq N+3}$ and implicitly depending also on the inverse string tension $\alpha^{\prime}$, are integrals on $N$ copies of the Riemann sphere $\mathbb{P}_{\mathbb{C}}^{1}$ of the form

$$
\begin{aligned}
& M_{N+3}(\boldsymbol{s}, \boldsymbol{n}, \tilde{\boldsymbol{n}})= \\
& =\int_{\left(\mathbb{P}_{\mathbb{C}}^{1}\right)} \prod_{i=1}^{N} d^{2} w_{i} \prod_{1 \leq i<j \leq N+2} e^{2 \alpha^{\prime} s_{i j} G^{P_{\mathbb{C}}^{1}}\left(w_{i}, w_{j}\right)}\left(\partial_{w_{j}} G^{P_{\mathbb{C}}^{1}}\left(w_{i}, w_{j}\right)\right)^{-2 n_{i j}}\left(\partial_{\bar{w}_{j}} G^{P_{\mathbb{C}}^{1}}\left(w_{i}, w_{j}\right)\right)^{-2 \tilde{n}_{i j}},
\end{aligned}
$$


where $w_{N+1}:=0, w_{N+2}:=1$ and $d^{2} w_{i}:=\frac{i d w_{i} d \bar{w}_{i}}{2 \pi}$. The Green function on the Riemann sphere is

$$
G^{P_{\mathrm{C}}^{1}}\left(z, z^{\prime}\right):=-\frac{1}{2} \log \left|z-z^{\prime}\right|^{2}
$$

so that the partial amplitudes reads

$$
M_{N+3}(\boldsymbol{s}, \boldsymbol{n}, \tilde{\boldsymbol{n}})=\int_{\left(\mathbb{P}_{\mathbb{C}}^{1}\right)} \prod_{i=1}^{N} d^{2} w_{i} \prod_{1 \leq i<j \leq N+2}\left|w_{i}-w_{j}\right|^{-2 \alpha^{\prime} s_{i j}}\left(w_{i}-w_{j}\right)^{n_{i j}}\left(\bar{w}_{i}-\bar{w}_{j}\right)^{\tilde{n}_{i j}}
$$

For instance, the Virasoro-Shapiro amplitude is a particular case with $N=1$ and vanishing integers $n_{i j}, \widetilde{n}_{i j}$ ?

$$
M_{4}(\boldsymbol{s}, \mathbf{0}, \mathbf{0})=\int_{\mathbb{P}_{\mathbb{C}}^{1}}|w|^{-2 \alpha^{\prime} s_{12}}|1-w|^{-2 \alpha^{\prime} s_{13}} d^{2} w=\frac{\Gamma\left(-\alpha^{\prime} s_{12}+1\right) \Gamma\left(-\alpha^{\prime} s_{13}+1\right) \Gamma\left(-\alpha^{\prime} s_{23}-1\right)}{\Gamma\left(\alpha^{\prime} s_{12}\right) \Gamma\left(\alpha^{\prime} s_{13}\right) \Gamma\left(\alpha^{\prime} s_{23}+2\right)} .
$$

\subsection{Open and unoriented tree-level amplitudes}

At tree level, open oriented (super)string amplitudes are defined over the disc and closed unoriented (super)string amplitudes are defined over the real projective plane.

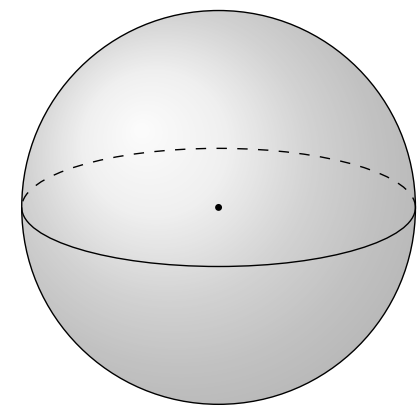

(a)

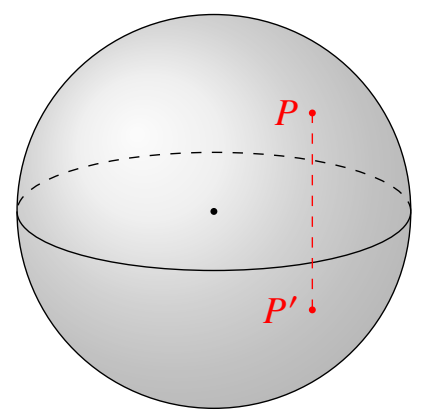

(b)

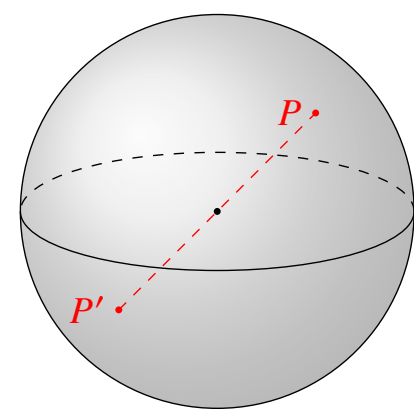

(c)

Figure 1.1: $(a)$ The Riemann sphere, $(b)$ the disc and $(c)$ the real projective plane. The disc and the real projective plane are obtained from the action of an involution identifying the points $P$ and $P^{\prime}$ on the Riemann sphere.

\subsubsection{The disc amplitudes}

The disc $\mathbb{D}_{2}$ is obtained by the action of the involution $\mathcal{I}^{\mathbb{D}_{2}}(z)=\bar{z}$ on the Riemann sphere: $\mathbb{D}_{2} \cong \mathbb{P}_{\mathbb{C}}^{1} / \mathcal{I}_{\mathbb{D}_{2}}$ (see figure 1.1(b)). The bosonic string propagator on the disc $\mathbb{D}_{2}$ is obtained from the method of images by applying equation (0.1), and reads

$$
G^{\mathbb{D}_{2}}\left(z, z^{\prime}\right)=-\frac{1}{2}\left(\log \left|z-z^{\prime}\right|^{2}+\log \left|\bar{z}-z^{\prime}\right|^{2}\right) .
$$

On the boundary of the disc where $z=x \in \mathbb{R}$ and $z^{\prime}=y \in \mathbb{R}$ we have

$$
G^{\mathbb{D}_{2}}(x, y)=-2 \log |x-y| .
$$


One can have all vertex operators inserted on the boundary of the disc. For instance, using the Green function above, identifying the boundary of the disc with $\mathbb{R}$ and fixing by conformal invariance three tachyon states at $0,1, \infty$, one obtains the celebrated Veneziano amplitude

$$
\int_{0}^{1} x^{-4 \alpha^{\prime} s_{12}}(1-x)^{-4 \alpha^{\prime} s_{13}} d x=\frac{\Gamma\left(-4 \alpha^{\prime} s_{12}+1\right) \Gamma\left(-4 \alpha^{\prime} s_{13}+1\right)}{\Gamma\left(2-4 \alpha^{\prime} s_{12}-4 \alpha^{\prime} s_{13}\right)} .
$$

More generally, four-point amplitudes of (open) bosonic strings involving both tachyons and massless states on the boundary of the disc differ from eq. (1.8) by a proportionality factor which is a rational function in the kinematic variables $\alpha^{\prime} s_{12}, \alpha^{\prime} s_{13}$.

One can also consider mixed amplitudes with insertion of $N_{o}$ open string external states on the boundary of the disc and $N_{c}$ closed string external states in the bulk of the disc. Such amplitudes were shown to be reducible to linear combinations of disc amplitudes with $N_{o}+2 N_{c}$ open string states on the boundary of the disc [42-44, 46].

As an illustration we present the case of the insertion of two states of momenta $k_{1}$ and $k_{2}$ at the respective position $z_{1}$ and $z_{2}$ in the bulk of the disc. The method of image on the covering plane doubles the insertions into two insertions at the positions $z_{1}$ and $\bar{z}_{1}$ and two insertions at the positions $z_{2}$ and $\bar{z}_{2}$. By conformal invariance we fix $z_{1}=0$ and $z_{2}=i x$ with $x \in[0,1]$. The integration over $z_{1}$ and $z_{2}$ is reduced to a single integration over $x \in[0,1]$. Using the Green function in (1.6) the two-point amplitude is then given by an integral of the type

$$
\int_{0}^{1}\left(x^{2}\right)^{-4 \alpha^{\prime} s_{12}-1}\left(1-x^{2}\right)^{-2 \alpha^{\prime} s_{13}} d x^{2}=-2 \alpha^{\prime} s_{13} \frac{\Gamma\left(-2 \alpha^{\prime} s_{12}\right) \Gamma\left(-2 \alpha^{\prime} s_{13}\right)}{\Gamma\left(1-2 \alpha^{\prime} s_{12}-2 \alpha^{\prime} s_{13}\right)}
$$

where $s_{12}=-\frac{1}{2} k_{1} \cdot k_{2}$ and $s_{13}=-\frac{1}{2} k_{1} \cdot D \cdot k_{1}$ and where $k_{i}$ is the momentum of the external state and $D \cdot k_{i}$ is the momentum of its image. Using the functional equation $\Gamma(x+1)=x \Gamma(x)$, we recognise that this amplitude only differs from the Veneziano amplitude in (1.8) just by a proportionality factor which is a rational function of the kinematic invariants (up to scaling the variables).

\subsubsection{The real projective plane amplitudes}

The action of the involution $\mathcal{I}_{\mathbb{P}_{\mathbb{R}}^{2}}(z)=-1 / \bar{z}$ on the Riemann sphere gives the real projective plane $\mathbb{P}_{\mathbb{R}}^{2} \cong \mathbb{P}_{\mathbb{C}}^{1} / \mathcal{I}_{\mathbb{P}_{\mathbb{R}}^{2}}$ (see figure 1.1(c)). The bosonic string propagator on the real projective plane $\mathbb{P}_{\mathbb{R}}^{2}$ is obtained from the method of images by applying equation (0.1), and reads

$$
G^{\mathbb{P}_{\mathrm{R}}^{2}}\left(z, z^{\prime}\right)=-\frac{1}{2}\left(\log \left|z-z^{\prime}\right|^{2}+\log \left|1+z^{\prime} \bar{z}\right|^{2}-\log \left|z z^{\prime}\right|^{2}\right) .
$$

The real projective plane does not have boundaries, so the amplitudes only involve closed string states. The amplitudes with $N_{c}$ closed string state insertions are, as in the previous section, reducible to linear combinations of disc amplitudes with $2 N_{c}$ open string states inserted on the boundary of the disc [45, 47, 48]. For instance, for the case of an amplitude with the insertion of two closed string states, the method of images on the covering plane doubles the insertions into two insertions at the positions $z_{1}$ and $\bar{z}_{1}$ and two insertions at the positions $z_{2}$ and $\bar{z}_{2}$. By conformal invariance we fix 
$z_{1}=0$ and $z_{2}=i x$ with $x \in[0,1]$. The integration over $z_{1}$ and $z_{2}$ is reduced to a single integration over $x \in[0,1]$. Using the Green function in (1.10) the two-point function is then given by an integral of the type

$$
\int_{0}^{1}\left(\left(x^{2}\right)^{-4 \alpha^{\prime} s_{12}-1}+\left(x^{2}\right)^{-4 \alpha^{\prime} s_{12}-4 \alpha^{\prime} s_{13}-1}\right)\left(1-x^{2}\right)^{-2 \alpha^{\prime} s_{13}} d x^{2}=-2 \alpha^{\prime} s_{13} \frac{\Gamma\left(-2 \alpha^{\prime} s_{12}\right) \Gamma\left(2 \alpha^{\prime} s_{13}+2 \alpha^{\prime} s_{13}\right)}{\Gamma\left(1+2 \alpha^{\prime} s_{13}\right)}
$$

where $s_{12}=-\frac{1}{2} k_{1} \cdot k_{2}$ and $s_{13}=-\frac{1}{2} k_{1} \cdot D \cdot k_{1}$ This amplitude differs from the Veneziano amplitude in (1.8) only by a proportionality factor which is a rational function of the kinematic invariants (up to scaling the variables).

We see on these examples that the main properties of the amplitude are not dictated by the local structure of the Green functions on the world-sheet, but by the boundary conditions on the integration space imposed by the action of the involution used to construct the open string geometry.

To summarize, all amplitudes on the disc or the real projective plane are reducible to open string amplitudes on the boundary of a disc

$$
A_{N+3}(\boldsymbol{s}, \boldsymbol{\epsilon})=\sum_{r, \rho} d_{r, \rho}(\boldsymbol{s}, \boldsymbol{\epsilon}) A_{N+3}\left(\boldsymbol{s}, \boldsymbol{n}^{r}, \rho\right)
$$

expanded on a basis of ordered integrals $A_{N+3}\left(\boldsymbol{s}, \boldsymbol{n}^{r}, \rho\right)$ along the real line, indexed by permutations $\rho \in \mathfrak{S}_{N}$, which we refer to as tree-level open string building blocks and we fix to be

$$
\begin{aligned}
A_{N+3}(\boldsymbol{s}, \boldsymbol{n}, \rho) & =\int_{x_{\rho(N)} \leq \cdots \leq x_{\rho(1)} \leq 0} \prod_{1 \leq m<n \leq N}\left(x_{\rho(n)}-x_{\rho(m)}\right)^{-4 \alpha^{\prime} s_{\rho(n) \rho(m)}+n_{\rho(n) \rho(m)}} \\
& \times \prod_{m=1}^{N}\left(-x_{\rho(m)}\right)^{-4 \alpha^{\prime} s_{N+1 \rho(m)}+n_{N+1 \rho(m)}}\left(1-x_{\rho(m)}\right)^{-4 \alpha^{\prime} s_{N+2 \rho(m)}+n_{N+2 \rho(m)}} \prod_{i=1}^{N} d x_{i} .
\end{aligned}
$$

Understanding the properties of any tree-level amplitudes in string theory is reduced to understand the building blocks (1.4) and (1.13) and their relations. This is the topic of the next two sections.

\section{Closed string amplitudes from single-valued correlation functions}

In this section we review the result of[38] that the KLT relation between the closed string building blocks in (1.4) and the open string building blocks in (1.12) is a special case of the conformal block decomposition of string theory correlation functions.

We introduce, for $\eta \in \mathbb{C}$ and $N \in \mathbb{N}$, the functions

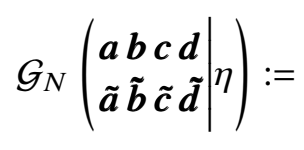




$$
\int_{\left(\mathbb{P}_{\mathbb{C}}^{1}\right)^{N}} \prod_{i=1}^{N} z_{i}^{a_{i}}\left(1-z_{i}\right)^{b_{i}}\left(\eta-z_{i}\right)^{c_{i}} \prod_{i=1}^{N} \bar{z}_{i}^{\tilde{a}_{i}}\left(1-\bar{z}_{i}\right)^{\tilde{b}_{i}}\left(\bar{\eta}-\bar{z}_{i}\right)^{\tilde{c}_{i}} \prod_{1 \leq i<j \leq N}\left(z_{i}-z_{j}\right)^{d_{i j}}\left(\bar{z}_{i}-\bar{z}_{j}\right)^{\tilde{d}_{i j}} \prod_{i=1}^{N} d^{2} z_{i},
$$

where the tuples of exponents $\boldsymbol{a}, \boldsymbol{b}, \boldsymbol{c}, \boldsymbol{d}, \tilde{\boldsymbol{a}}, \tilde{\boldsymbol{b}}, \tilde{\boldsymbol{c}}, \tilde{\boldsymbol{d}}$ are formed by complex numbers which satisfy

$$
\begin{array}{rr}
a_{i}-\tilde{a}_{i}, b_{i}-\tilde{b}_{i}, c_{i}-\tilde{c}_{i}, \in \mathbb{Z} & 1 \leq i \leq N, \\
d_{i j}-\tilde{d}_{i j} \in \mathbb{Z} & 1 \leq i<j \leq N .
\end{array}
$$

These conditions imply that the integrand of (2.1) is single-valued and therefore that the integral over $\mathbb{C}^{n}$ makes sense and defines a single-valued function of $\eta \in \mathbb{C} \backslash\{0,1\}$ for those exponents such that the integral converges absolutely.

The partial amplitudes (1.4) are the values at $\eta=1$ of the functions (2.1) when the exponents satisfy the relations

$$
\begin{array}{rlrl}
a_{i}:=-\alpha^{\prime} s_{i N+1}+n_{i N+1}, & b_{i}+c_{i}:=-\alpha^{\prime} s_{i N+2}+n_{i N+2}, & 1 \leq i \leq N, \\
\tilde{a}_{i}:=-\alpha^{\prime} s_{i N+1}+\tilde{n}_{i N+1}, & \tilde{b}_{i}+\tilde{c}_{i}:=-\alpha^{\prime} s_{i N+2}+\tilde{n}_{i N+2}, & 1 \leq i \leq N, \\
d_{i j}:=-\alpha^{\prime} s_{i j}+n_{i j}, \quad \tilde{d}_{i j}=-\alpha^{\prime} s_{i j}+\tilde{n}_{i j}, & 1 \leq i<j \leq N .
\end{array}
$$

Following the standard rules for the correlation functions of conformal field theory minimal models [39] and string theory in $[18,19,67,68]$, one can express the function $\mathcal{G}_{N}$ as a conformal correlation function:

$$
\mathcal{G}_{N}\left(\begin{array}{c}
\boldsymbol{a} \boldsymbol{b} \boldsymbol{c} \boldsymbol{d} \\
\tilde{\boldsymbol{a}} \tilde{\boldsymbol{b}} \tilde{\boldsymbol{c}} \tilde{\boldsymbol{d}}
\end{array} \mid \eta\right)=\int_{\left(\mathbb{P}_{\mathbb{C}}^{1}\right)^{N}} \prod_{i=1}^{N} d^{2} z_{i}\left\langle V_{0}(0) \prod_{i=1}^{N} V_{i}\left(z_{i}\right) V_{N+1}(1) V_{N+2}(\infty) U(\eta)\right\rangle
$$

with $U(\eta)=: \exp \left(i k_{*} \cdot X(\eta)\right)$ : an auxiliary vertex operator. The fact that $\mathcal{G}_{N}$ is single-valued is coherent with the axioms of conformal field theory, because it guarantees the locality of the theory.

\subsection{Holomorphic factorisation}

It was shown in [38] that the correlation function (2.1) has the holomorphic factorisation

$$
\mathcal{G}_{N}\left(\begin{array}{c}
\boldsymbol{a} \boldsymbol{b} \boldsymbol{c} \boldsymbol{d} \\
\tilde{\boldsymbol{a}} \tilde{\boldsymbol{b}} \tilde{\boldsymbol{c}} \tilde{\boldsymbol{d}}
\end{array} \mid \eta\right)=\frac{1}{\pi^{N}} \vec{J}_{N}(\tilde{\boldsymbol{a}}, \tilde{\boldsymbol{b}}, \tilde{\boldsymbol{c}} ; \tilde{\boldsymbol{d}} ; \bar{\eta})^{T} \hat{G}_{N}(\boldsymbol{a}, \boldsymbol{b}, \boldsymbol{c} ; \boldsymbol{d}) \vec{J}_{N}(\boldsymbol{a}, \boldsymbol{b}, \boldsymbol{c} ; \boldsymbol{d} ; \eta)
$$

with

(i) The vector $\vec{J}_{N}(\boldsymbol{a}, \boldsymbol{b}, \boldsymbol{c} ; \boldsymbol{d} ; \eta):=\left\{J_{(\sigma, \rho)}(\boldsymbol{a}, \boldsymbol{b}, \boldsymbol{c} ; \boldsymbol{d} ; \eta),(\rho, \sigma) \in \mathfrak{S}_{r} \times \mathfrak{S}_{s}, r+s=N\right\}$ of AomotoGel'fand hypergeometric functions [69-71], i.e. the iterated integrals

$$
J_{(\rho, \sigma)}(\boldsymbol{a}, \boldsymbol{b}, \boldsymbol{c} ; \boldsymbol{d} ; \eta)=\int_{\tilde{\Delta}_{(\rho, \sigma)}(\eta)} \prod_{m=1}^{r} \prod_{n=1}^{s}\left(z_{\sigma(n)}-z_{\rho(m)}\right)^{d_{\rho(m) \sigma(n)}} \prod_{1 \leq m<n \leq r}\left(z_{\rho(m)}-z_{\rho(n)}\right)^{d_{\rho(m) \rho(n)}}
$$




$$
\begin{array}{r}
\times \prod_{1 \leq m<n \leq s}\left(z_{\sigma(m)}-z_{\sigma(n)}\right)^{d_{\sigma(m) \sigma(n)}} \prod_{m=1}^{r}\left(-z_{\rho(m)}\right)^{a_{\rho(m)}}\left(1-z_{\rho(m)}\right)^{b_{\rho(m)}}\left(\eta-z_{\rho(m)}\right)^{c_{\rho(m)}} \\
\times \prod_{n=1}^{s}\left(z_{\sigma(n)}\right)^{a_{\sigma(n)}}\left(1-z_{\sigma(n)}\right)^{b_{\sigma(n)}}\left(z_{\sigma(n)}-\eta\right)^{c_{\sigma(n)}} \prod_{i=1}^{N} d z_{i}, \quad
\end{array}
$$

integrated over the domain

$$
\tilde{\Delta}_{(\rho, \sigma)}(\eta):=\left\{z_{\rho(r)} \leq \cdots \leq z_{\rho(1)} \leq 0 \leq \eta \leq z_{\sigma(s)} \leq \cdots \leq z_{\sigma(1)} \leq 1\right\}
$$

with $(\rho, \sigma) \in \mathfrak{S}_{r} \times \mathfrak{S}_{s}$ and $(\rho, \sigma) \in \mathfrak{S}_{r} \times \mathfrak{S}_{k}$ the set of permutation of $k$-elements.

(ii) $\hat{G}_{N}(\boldsymbol{a}, \boldsymbol{b}, \boldsymbol{c} ; \boldsymbol{d})$ is an invertible square matrix of size $(N+1)$ !, independent of $\eta$, whose entries are rational linear combinations of exponentials of $\mathbb{Q}(\pi i)$-linear combinations of the parameters $a_{i}, b_{i}, c_{i}$ and $d_{i j}$

The relation (2.5) is the usual holomorphic factorisation of tree-level conformal correlation function on conformal blocks. The conformal blocks are the mutivalued integrals (2.6).

\subsubsection{The $N=1$ case}

We illustrate how the factorisation works in the $N=1$ case corresponding to the four-point amplitude. We want to perform the holomorphic factorisation of the integral

$$
\mathcal{G}_{1}\left(\begin{array}{ccc}
a & b & c \\
\tilde{a} & \tilde{b} & \tilde{c}
\end{array} \mid \eta\right)=\int_{\mathbb{P}_{\mathbb{C}}^{1}} z^{a} \bar{z}^{\tilde{a}}(z-1)^{b}(\bar{z}-1)^{\tilde{b}}(z-\eta)^{c}(\bar{z}-\bar{\eta})^{\tilde{c}} d^{2} z
$$

with the integer spin conditions

$$
a-\tilde{a} \in \mathbb{Z}, \quad b-\tilde{b} \in \mathbb{Z}, \quad c-\tilde{c} \in \mathbb{Z} .
$$

Following the notation of (2.6) we have

$$
\vec{J}_{1}(a, b, c ; \eta)=\left(\begin{array}{l}
J_{(I d, \emptyset)}(a, b, c ; \eta) \\
J_{(\emptyset, I d)}(a, b, c ; \eta)
\end{array}\right)=\left(\begin{array}{c}
\int_{-\infty}^{0}(-z)^{a}(1-z)^{b}(\eta-z)^{c} d z \\
\int_{\eta}^{1} z^{a}(1-z)^{b}(z-\eta)^{c} d z
\end{array}\right)
$$

The conformal block decomposition gives

$$
\mathcal{G}_{1}\left(\begin{array}{ccc}
a & b & c \\
\tilde{a} & \tilde{b} & \tilde{c}
\end{array} \mid \eta\right)=\frac{1}{\pi} \vec{J}_{1}(\tilde{a}, \tilde{b}, \tilde{c} ; \bar{\eta})^{T} \hat{G}_{1}(a, b, c) \vec{J}_{1}(a, b, c ; \eta)
$$

with

$$
\hat{G}_{1}(a, b, c)=\frac{-1}{\sin (\pi(b+c))}\left(\begin{array}{cc}
\sin (\pi(a+b+c)) \sin (\pi a) & 0 \\
0 & \sin (\pi b) \sin (\pi c)
\end{array}\right)
$$




\subsection{Closed string amplitudes}

Setting $\eta=1$ in the holomorphic factorisation leads to a fairly simple expression, because at $\eta=1$ only the first $N$ ! rows $\vec{J}_{N}$ of the vector of Aomoto-Gel'fand hypergeometric functions $\vec{J}_{N}$ in (2.6) are non-vanishing: ${ }^{1}$

$$
\vec{J}_{N}(\boldsymbol{a}, \boldsymbol{b}, \boldsymbol{c} ; \boldsymbol{d} ; 1)=\left(\begin{array}{c}
\vec{J}_{N}(\boldsymbol{a}, \boldsymbol{b}, \boldsymbol{c} ; \boldsymbol{d}) \\
0
\end{array}\right)
$$

where

$\vec{J}(\rho, \sigma)(\boldsymbol{a}, \boldsymbol{b}, \boldsymbol{c} ; \boldsymbol{d})=\int_{\tilde{\delta}_{(\rho, \sigma)}} \prod_{1 \leq m<n \leq N}\left(z_{\rho(m)}-z_{\rho(n)}\right)^{d_{\rho(m) \rho(n)}} \prod_{m=1}^{N}\left(-z_{\rho(m)}\right)^{a_{\rho(m)}}\left(1-z_{\rho(m)}\right)^{b_{\rho(m)}+c_{\rho(m)}} \prod_{i=1}^{N} d z_{i}$,

integrated over the domain

$$
\tilde{\delta}_{(\rho, \sigma)}:=\left\{z_{\rho(N)} \leq \cdots \leq z_{\rho(1)} \leq 0\right\}
$$

These integrals can be identified with the open string building blocks $A_{N+3}^{\rho}(\boldsymbol{s}, \boldsymbol{n})$ of (1.13).

Using the parameter identification (2.3) and the holomorphic factorization, we find

$$
M_{N+3}(\boldsymbol{s}, \boldsymbol{n}, \tilde{\boldsymbol{n}})=\mathcal{G}_{N}\left(\begin{array}{l}
\boldsymbol{a} \boldsymbol{b} \boldsymbol{c} \boldsymbol{d} \\
\tilde{\boldsymbol{a}} \tilde{\boldsymbol{b}} \tilde{\boldsymbol{c}} \tilde{\boldsymbol{d}}
\end{array} \mid 1\right)=\frac{1}{\pi^{N}} \vec{\jmath}_{N}(\tilde{\boldsymbol{a}}, \tilde{\boldsymbol{b}}, \tilde{\boldsymbol{c}} ; \boldsymbol{d})^{T} \hat{G}_{N}^{(1)}(\boldsymbol{a}, \boldsymbol{b}, \boldsymbol{c} ; \boldsymbol{d}) \vec{\jmath}_{N}(\boldsymbol{a}, \boldsymbol{b}, \boldsymbol{c} ; \boldsymbol{d}),
$$

where $\hat{G}_{N}^{(1)}(\boldsymbol{a}, \boldsymbol{b}, \boldsymbol{c} ; \boldsymbol{d})$ is the upper-left $N \times N$ block matrix contained in $\hat{G}_{N}(\boldsymbol{a}, \boldsymbol{b}, \boldsymbol{c} ; \boldsymbol{d})$.

This approach leads to an expression of the closed string building blocks in terms of the size $N$ ! vector $\vec{J}_{N}(\boldsymbol{a}, \boldsymbol{b}, \boldsymbol{c} ; \boldsymbol{d})$ of open string building blocks and the matrix $\hat{G}_{N}^{(1)}$, which is a non-local version of the KLT relations given in [49, 72]. One finds back the usual KLT relations [29] using the monodromy relations $[46,73]$ between open string ordered integrals.

Although the end result for the closed string building block only depends on $\hat{G}_{N}^{(1)}$, it is necessary to have the full matrix $\hat{G}_{N}$ to get rid of the monodromies when $\eta$ varies.

\subsubsection{The four-point case}

We illustrate the previous results on the $N=1$ case corresponding to the four-point amplitude

$$
M_{4}(\boldsymbol{s}, \boldsymbol{n}, \tilde{\boldsymbol{n}})=\int_{\mathbb{P}_{\mathbb{C}}}|z|^{-2 \alpha^{\prime} s_{12}} z^{n_{12}} \bar{z}^{\tilde{n}_{12}}|1-z|^{-2 \alpha^{\prime} s_{13}}(1-z)^{n_{13}}(1-\bar{z})^{\tilde{n}_{13}} d^{2} z
$$

We can write

$$
M_{4}(\boldsymbol{s}, \boldsymbol{n}, \tilde{\boldsymbol{n}})=\mathcal{G}_{1}\left(\begin{array}{ccc}
a & b & c \\
\tilde{a} & \tilde{b} & \tilde{c}
\end{array} \mid 1\right)
$$

${ }^{1}$ The lower $N \times N$ ! components vanish whenever the integral is absolutely convergent. This leads to (2.13) by analytic continuation on the parameters $\boldsymbol{b}, \boldsymbol{c}$ and $\boldsymbol{d}$. 
with the identification of the parameters $a=-\alpha^{\prime} s_{12}+n_{12}, \tilde{a}=-\alpha^{\prime} s_{12}+\tilde{n}_{12}, b+c=-\alpha^{\prime} s_{13}+n_{13}$ and $\tilde{b}+\tilde{c}=-\alpha^{\prime} s_{13}+\tilde{n}_{13}$. The second component of $\vec{J}_{1}(a, b, c ; \eta)$ in (2.10) vanishes as $\eta \rightarrow 1$ in the convergence region, and therefore everywhere by analytic continuation.

With such identifications, $j_{1}(a, b, c)$ is nothing but the integral

$$
A(1234 ; \boldsymbol{s}, \boldsymbol{n}):=\int_{-\infty}^{0}(-z)^{-\alpha^{\prime} s_{12}+n_{12}}(1-z)^{-\alpha^{\prime} s_{13}+n_{13}} d z
$$

where (1234) refers to the permutation of the points $z, 0,1,+\infty$ on the real line which gives the ordered integral (2.19). The holomorphic decomposition in (2.11) evaluated at $\eta=1$ then gives (using the momentum conservation condition $s_{12}+s_{13}+s_{23}=0$ )

$$
M_{4}(\boldsymbol{s}, \boldsymbol{n}, \tilde{\boldsymbol{n}})=-\frac{1}{\pi} \frac{\sin \left(\alpha^{\prime} \pi s_{12}\right) \sin \left(\alpha^{\prime} \pi s_{23}\right)}{\sin \left(\alpha^{\prime} \pi s_{13}\right)} A(1234 ; \boldsymbol{s}, \boldsymbol{n}) A(1234 ; \boldsymbol{s}, \tilde{\boldsymbol{n}}) .
$$

The KLT relation with the inverse momentum kernel arises from this formalism. The ordered integral $A(1234 ; \boldsymbol{s}, \boldsymbol{n})$ is just one of the possible four-point open string ordered integrals, but by standard monodromy relations $[46,73]$ any other four-point ordered integral must be related to $A(1234 ; \boldsymbol{s}, \boldsymbol{n})$. For instance, if we consider the permutation (2134) corresponding to the integral

$$
A(2134 ; \boldsymbol{s}, \boldsymbol{n}):=\int_{0}^{1} z^{-\alpha^{\prime} s_{12}+n_{12}}(1-z)^{-\alpha^{\prime} s_{13}+n_{13}} d z
$$

we have the monodromy relation

$$
\sin \left(\alpha^{\prime} \pi s_{23}\right) A(1234 ; \boldsymbol{s}, \boldsymbol{n})=\sin \left(\alpha^{\prime} \pi s_{13}\right) A(2134 ; \boldsymbol{s}, \boldsymbol{n}) .
$$

Plugging this relation in (2.20) we recover ${ }^{2}$ the KLT expression for the closed string four-point amplitudes given in [29, eq. (3.11)]:

$$
\begin{aligned}
M_{4}(\boldsymbol{s}, \boldsymbol{n}, \tilde{\boldsymbol{n}}) & =-\frac{1}{\pi} \sin \left(\alpha^{\prime} \pi s_{12}\right) A(1234 ; \boldsymbol{s}, \boldsymbol{n}) A(2134 ; \boldsymbol{s}, \tilde{\boldsymbol{n}}) \\
& =-\frac{1}{\pi} \sin \left(\alpha^{\prime} \pi s_{12}\right) A(2134 ; \boldsymbol{s}, \boldsymbol{n}) A(1234 ; \boldsymbol{s}, \tilde{\boldsymbol{n}}) .
\end{aligned}
$$

This illustrates how the momentum kernel $\mathcal{S}_{N}$ from [74] arises as the product of the holomorphic factorisation matrix $\hat{G}_{N}^{(1)}$ times the change of basis matrix between the ordered open string integrals:

$$
\mathcal{S}_{1}\left(\alpha^{\prime} s_{12}\right)=-\sin \left(\alpha^{\prime} \pi s_{12}\right)=-\frac{\sin \left(\alpha^{\prime} \pi s_{12}\right) \sin \left(\alpha^{\prime} \pi s_{23}\right)}{\sin \left(\alpha^{\prime} \pi s_{13}\right)} \times \frac{\sin \left(\alpha^{\prime} \pi s_{13}\right)}{\sin \left(\alpha^{\prime} \pi s_{23}\right)} .
$$

The $\Gamma$-function representation of the (beta-) integrals defining $A(1234 ; \boldsymbol{s}, \boldsymbol{n})$ and $A(2134 ; \boldsymbol{s}, \boldsymbol{n})$ is

$$
A(1234 ; \boldsymbol{s}, \tilde{\boldsymbol{n}})=\frac{\Gamma\left(1-\alpha^{\prime} s_{12}+\tilde{n}_{12}\right) \Gamma\left(-1-\alpha^{\prime} s_{23}-\tilde{n}_{12}-\tilde{n}_{13}\right)}{\Gamma\left(\alpha^{\prime} s_{13}-\tilde{n}_{13}\right)}
$$

${ }^{2}$ The sign difference is given by a different notation for the Mandelstam variables. 


$$
A(2134 ; \boldsymbol{s}, \boldsymbol{n})=\frac{\Gamma\left(1-\alpha^{\prime} s_{12}+n_{12}\right) \Gamma\left(1-\alpha^{\prime} s_{13}+n_{13}\right)}{\Gamma\left(2+\alpha^{\prime} s_{23}+n_{12}+n_{13}\right)}
$$

Using the momentum conservation condition $s_{12}+s_{13}+s_{23}=0$ and Euler's reflection formula $\Gamma(x) \Gamma(1-x)=\frac{\pi}{\sin (\pi x)}$, the four-point partial amplitude reads

$$
M_{4}(\boldsymbol{s}, \boldsymbol{n}, \tilde{\boldsymbol{n}})=-(-1)^{\tilde{n}_{13}} \frac{\Gamma\left(1-\alpha^{\prime} s_{12}+n_{12}\right)}{\Gamma\left(\alpha^{\prime} s_{12}-\tilde{n}_{12}\right)} \frac{\Gamma\left(1-\alpha^{\prime} s_{13}+n_{13}\right)}{\Gamma\left(\alpha^{\prime} s_{13}-\tilde{n}_{13}\right)} \frac{\Gamma\left(-1-\alpha^{\prime} s_{23}-\tilde{n}_{12}-\tilde{n}_{13}\right)}{\Gamma\left(2+\alpha^{\prime} s_{23}+n_{12}+n_{13}\right)}
$$

Using repeatedly that $\Gamma(1+x)=x \Gamma(x)$, one can find a rational function $Q\left(\alpha^{\prime} s_{12}, \alpha^{\prime} s_{13}, \alpha^{\prime} s_{23}\right)$ with integer coefficients (depending on the integer parameters $n_{12}, n_{13}, \tilde{n}_{12}, \tilde{n}_{13}$ ) such that

$$
M_{4}(\boldsymbol{s}, \boldsymbol{n}, \tilde{\boldsymbol{n}})=Q\left(\alpha^{\prime} s_{12}, \alpha^{\prime} s_{13}, \alpha^{\prime} s_{23}\right) \frac{\Gamma\left(1-\alpha^{\prime} s_{12}\right)}{\Gamma\left(1+\alpha^{\prime} s_{12}\right)} \frac{\Gamma\left(1-\alpha^{\prime} s_{13}\right)}{\Gamma\left(1+\alpha^{\prime} s_{13}\right)} \frac{\Gamma\left(1-\alpha^{\prime} s_{23}\right)}{\Gamma\left(1+\alpha^{\prime} s_{23}\right)} \text {. }
$$

Euler's formula

$$
\Gamma(1+x)=e^{-\gamma x} \exp \left(\sum_{m \geq 2} \frac{\zeta(m)}{m}(-x)^{m}\right),
$$

where $\gamma$ is the Euler-Mascheroni constant, implies that

$$
\frac{\Gamma(1+x)}{\Gamma(1-x)}=e^{-2 \gamma x} \exp \left(-2 \sum_{m=1}^{\infty} \frac{\zeta(2 m+1)}{2 m+1} x^{2 m+1}\right)
$$

Therefore, by momentum conservation, the four-point partial amplitude takes the form

$M_{4}(\boldsymbol{s}, \boldsymbol{n}, \tilde{\boldsymbol{n}})=Q\left(\alpha^{\prime} s_{12}, \alpha^{\prime} s_{13}, \alpha^{\prime} s_{23}\right) \exp \left(2 \sum_{m=1}^{\infty} \frac{\zeta(2 m+1)}{2 m+1}\left(\left(\alpha^{\prime} s_{12}\right)^{2 m+1}+\left(\alpha^{\prime} s_{13}\right)^{2 m+1}+\left(\alpha^{\prime} s_{23}\right)^{2 m+1}\right)\right)$.

The argument of the exponential factor only involves odd Riemann zeta values. As we will see in the next section, the fact that even zeta values do not appear is part of a more general pattern which seems to constitute a fundamental feature of closed string theories.

\section{Small $\alpha^{\prime}$ expansion of tree-level building blocks}

The disc amplitude building blocks with $N+3$ external states on the boundary (1.12) can be reduced (rescaling $\alpha^{\prime}$ by a factor of 4 ) to the finite set of integrals [57]

$$
Z_{\rho, \sigma}^{(N)}\left(\alpha^{\prime} \boldsymbol{s}\right):=\int_{0 \leq x_{\sigma(1)} \leq \cdots \leq x_{\sigma(N)} \leq 1} \frac{\prod_{1 \leq i<j \leq N+2}\left|x_{i}-x_{j}\right|^{-\alpha^{\prime} s_{i j}}}{x_{\rho(1)}\left(1-x_{\rho(N)}\right) \prod_{i=2}^{N}\left(x_{\rho(i)}-x_{\rho(i-1)}\right)} \prod_{i=1}^{N} d x_{i}
$$


with $\rho, \sigma \in \mathfrak{S}_{N}$ permutations of $N$ letters, $x_{N+1}:=0, x_{N+2}:=1$. Similarly, the closed string amplitude building blocks in (1.4) can be expanded on the finite set of integrals $[55,75,76]$

$J_{\rho, \sigma}^{(N)}\left(\alpha^{\prime} \boldsymbol{s}\right):=\int_{\left(\mathbb{P}_{\mathbb{C}}^{1}\right)^{N}} \frac{\prod_{1 \leq i<j \leq N+2}\left|z_{i}-z_{j}\right|^{-2 \alpha^{\prime} s_{i j}}}{z_{\rho(1)} \bar{z}_{\sigma(1)}\left(1-z_{\rho(N)}\right)\left(1-\bar{z}_{\sigma(N)}\right) \prod_{i=2}^{N}\left(z_{\rho(i)}-z_{\rho(i-1)}\right)\left(\bar{z}_{\sigma(i)}-\bar{z}_{\sigma(i-1)}\right)} \prod_{i=1}^{N} d^{2} z_{i}$,

where $d^{2} z_{i}:=i d z d \bar{z} / 2 \pi, z_{N+1}:=0, z_{N+2}:=1$.

It was shown in [76] that $Z_{\rho, \sigma}^{(N)}\left(\alpha^{\prime} s\right)$ have a Laurent series expansion as $\alpha^{\prime}$ tends to zero, with coefficients belonging to the ring $\mathcal{Z}$ of rational linear combination of multiple zeta values, the real numbers defined for $k_{1}, \ldots, k_{r} \in \mathbb{N}, k_{r} \geq 2$, by the absolutely convergent series

$$
\zeta\left(k_{1}, \ldots, k_{r}\right):=\sum_{0<n_{1}<\cdots<n_{r}} \frac{1}{n_{1}^{k_{1}} \cdots n_{r}^{k_{r}}} .
$$

The functions $J_{\rho, \sigma}^{(N)}\left(\alpha^{\prime} s_{i j}\right)$ also have a Laurent series expansion as $\alpha^{\prime}$ tends to zero, and extensive computations led to conjecture in [54] that the coefficients belong to a subring $\mathcal{Z}^{\text {sv }}$ of multiple zeta values, called the ring of single-valued multiple zeta values, which we will introduce in section 3.1 below. This conjecture was recently proven, independently and in three different ways, in the papers [50, 57] and in our paper [38].

The methods of [50,57] lead to proving a stronger statement, which relates $J_{\rho, \sigma}^{(N)}\left(\alpha^{\prime} s_{i j}\right)$ to $Z_{\rho, \sigma}^{(N)}\left(\alpha^{\prime} s_{i j}\right)$ via the single-valued projection, a surjective map from the (motivic) ring of multiple zeta values to the (motivic) ring of single-valued multiple zeta values. Both of these proofs can be used to explicitly compute the $\alpha^{\prime}$-expansion of $J_{\rho, \sigma}^{(N)}\left(\alpha^{\prime} s_{i j}\right)$ provided that we know the $\alpha^{\prime}$-expansion of $Z_{\rho, \sigma}^{(N)}\left(\alpha^{\prime} s_{i j}\right)$.

Our proof, instead, does not involve open string integrals, and can be used to algorithmically compute the coefficients of $J_{\rho, \sigma}^{(N)}\left(\alpha^{\prime} s_{i j}\right)$ directly from their definition. In section 3.2 we give a detailed explanation of our approach in the simplest case where $N=1$. An advantage of our method is that it allows to compute a broad class of multiple complex integrals in terms of single-valued multiple zeta values. For instance, we will see in section 4.2 that one can use the same techniques to prove that certain asymptotic limits of genus-one closed string amplitudes involve single-valued multiple zeta values.

\subsection{Single-valued multiple zeta values}

We consider the alphabet $X=\left\{x_{0}, x_{1}\right\}$ formed by two formal non-commutative letters $x_{0}$ and $x_{1}$, and we denote by $X^{*}$ the set of all possible words $w=x_{i_{1}} \cdots x_{i_{n}}$ in this alphabet. We fix a simply connected complex domain $U$ obtained by removing from $\mathbb{C}$ two non-intersecting half-lines (the branch cuts) connecting $\infty$ with 0 and 1 , respectively.

To every word $w \in X^{*}$ we associate a multiple polylogarithm (in one variable) $L_{w}(z)$, the holomorphic function of $z \in U$ defined recursively by setting $L_{x_{0}^{r}}(z):=(\log (z))^{r} / r$ ! (the principal 
branch on $U$ ) for a string $x_{0}^{r}$ of $r$ consecutives $x_{0}$ 's and by setting for any other word $w=x_{i_{1}} \cdots x_{i_{n}} \in X^{*}$

$$
L_{w}(z):=\int_{[0, z]} \frac{d z^{\prime}}{z^{\prime}-i_{1}} L_{x_{i_{2}} \cdots x_{i_{n}}}\left(z^{\prime}\right)
$$

where $[0, z]$ is any path in $U$. These functions are well-defined, because they are invariant under deformations of the path inside the same homotopy class, and thus depend only on the endpoint $z$, because the fundamental group of $U$ is trivial. They can be extended to multi-valued functions on $\mathbb{C} \backslash\{0,1\}$, whose values depend on $z$ and on the homotopy class of the path $[0, z]$ inside $\mathbb{C} \backslash\{0,1\}$.

For all $w$ there exist an integer $K_{0} \geq 0$ and holomorphic functions $f_{0}, \ldots, f_{K_{0}}$ vanishing at 0 such that, if $z \in U$ is close enough to 0 , then

$$
L_{w}(z)=\sum_{k=0}^{K_{0}} \frac{(\log (z))^{k}}{k !} f_{k}(z) .
$$

Moreover, for all $w$ there exist an integer $K_{1} \geq 0$ and holomorphic functions $g_{0}, \ldots, g_{K_{1}}$ whose values at 1 belong to the ring $\mathcal{Z}$ of multiple zeta values defined by (3.3) such that, if $z \in U$ is close enough to 1 , then

$$
L_{w}(z)=\sum_{k=0}^{K_{1}} \frac{(\log (1-z))^{k}}{k !} g_{k}(z) .
$$

We define the regularised special values $L_{w}(1):=g_{0}(1) \in \mathcal{Z}$ (which coincide with the limit at $z=1$ if the word $w$ starts with $\left.x_{0}\right)$. In particular, $\zeta\left(k_{1}, \ldots, k_{r}\right)=(-1)^{r} L_{x_{0}^{k_{r}-1} x_{1} \cdots x_{0}^{k_{1}-1} x_{1}}(1)$.

The monodromies of multiple polylogarithms along paths in $\mathbb{C} \backslash\{0,1\}$ crossing the branch cuts are best described in terms of the generating series

$$
L_{\left\{x_{0}, x_{1}\right\}}(z):=\sum_{w \in X^{*}} L_{w}(z) \cdot w .
$$

This is, up to constants, the unique holomorphic $\mathbb{C}\langle\langle X\rangle\rangle$-valued solution in $U$ of the KnizhnikZamolodchikov (KZ) equation

$$
\frac{\partial}{\partial z} F(z)=\left(\frac{x_{0}}{z}+\frac{x_{1}}{z-1}\right) F(z)
$$

such that $F(z)=\exp \left(x_{0} \log (z)\right) f(z)$ as $z \rightarrow 0$, with $f$ holomorphic $\mathbb{C}\langle\langle X\rangle\rangle$-valued such that $f(0)=1$. The regularised special value $Z_{\left\{x_{0}, x_{1}\right\}}:=L_{\left\{x_{0}, x_{1}\right\}}(1)$, i.e. the generating series of (regularised) multiple zeta values, is known as the Drinfel'd associator. With this notation, the action of the monodromy operators $M_{0}$ and $M_{1}$ (defined by analytic continuation along loops winding once counterclockwise around 0 and 1 , respectively) is given by

$$
M_{0} L_{\left\{x_{0}, x_{1}\right\}}(z)=L_{\left\{x_{0}, x_{1}\right\}}(z) e^{2 \pi i x_{0}}, \quad M_{1} L_{\left\{x_{0}, x_{1}\right\}}(z)=L_{\left\{x_{0}, x_{1}\right\}}(z) Z_{\left\{x_{0}, x_{1}\right\}}^{-1} e^{2 \pi i x_{1}} Z_{\left\{x_{0}, x_{1}\right\}}
$$

Looking at the monodromy around $z=0$, a first step towards constructing single-valued analogues of multiple polylogarithms is to define on $X^{*}$ the operator $\widehat{x_{i_{1}} \cdots x_{i_{n}}}:=x_{i_{n}} \cdots x_{i_{1}}$, extend it by linearity 
to $\mathbb{C}\langle\langle X\rangle\rangle$ and consider the product $L_{\left\{x_{0}, x_{1}\right\}}(z) \overline{\overline{L_{\left\{x_{0}, x_{1}\right\}}(z)}}$. This new function is now single-valued around $z=0$, but it is still multi-valued around $z=1$. It turns out [77] that one can "deform" the letter $x_{1}$ to a new letter $y\left(x_{0}, x_{1}\right)=x_{1}+\ldots \in \mathcal{Z}\langle\langle X\rangle\rangle$ so that the new function

$$
\mathcal{L}_{\left\{x_{0}, x_{1}\right\}}(z):=L_{\left\{x_{0}, x_{1}\right\}}(z) \overline{\overline{L_{\left\{x_{0}, y\left(x_{0}, x_{1}\right)\right\}}(z)}}
$$

is single-valued (but no-longer holomorphic) on the punctured complex plane $\mathbb{C} \backslash\{0,1\}$. It can be seen as a generating function

$$
\mathcal{L}_{\left\{x_{0}, x_{1}\right\}}(z)=: \sum_{w \in X^{*}} \mathcal{L}_{w}(z) \cdot w
$$

of single-valued smooth functions $\mathcal{L}_{w}(z)$, which are called single-valued multiple polylogarithms, whose simplest examples $\mathcal{L}_{x_{0}^{r}}(z)=\left(\log |z|^{2}\right)^{r} / r !$ are indeed "single-valued versions" of the corresponding multiple polylogarithms $L_{x_{0}^{r}}(z)=(\log (z))^{r} / r$ !. Remarkably, also the function $\mathcal{L}_{\left\{x_{0}, x_{1}\right\}}(z)$ is a solution to the $\mathrm{KZ}$ equation, and the map $L_{w}(z) \rightarrow \mathcal{L}_{w}(z)$ respects shuffle product identities.

Single-valued multiple zeta values are defined as the (regularised) special values $\mathcal{L}_{w}(1)$ obtained by setting $z=1$ in the equation (3.10). Because $y\left(x_{0}, x_{1}\right) \in \mathcal{Z}\langle\langle X\rangle\rangle$, they also belong to $\mathcal{Z}$, but in fact they form a (conjecturally) much smaller subring $\mathcal{Z}^{\mathrm{sv}}$. Assuming standard conjectures, the map $L_{w}(z) \rightarrow \mathcal{L}_{w}(z)$ specializes at $z=1$ to a surjective map of rings $\mathcal{Z} \rightarrow \mathcal{Z}^{\text {sv }}$, which we call the "singlevalued projection". It therefore seems natural to define $\zeta^{\mathrm{sv}}\left(k_{1}, \ldots, k_{r}\right):=(-1)^{r} \mathcal{L}_{x_{0}^{k_{r}-1} x_{1} \cdots x_{0}^{k_{1}-1} x_{1}}(1)$, and one finds for instance $\zeta^{\mathrm{sv}}(2 k)=0$ and $\zeta^{\mathrm{sv}}(2 k+1)=2 \zeta(2 k+1)$.

\subsection{The $\alpha^{\prime}$-expansion of the four-point amplitude from single-valued integration}

We illustrate the approach of [38] to prove that the coefficients of tree-level closed string building blocks are single-valued multiple zeta values in the case of the four-point integral

$$
V(s, t):=\int_{\mathbb{P}_{\mathbb{C}}}|z|^{2 s-2}|1-z|^{2 t-2} d^{2} z .
$$

This function coincides with the Virasoro-Shapiro amplitude (1.5) upon setting $s:=-\alpha^{\prime} s_{12}+1$ and $t:=-\alpha^{\prime} s_{13}+1$ and coincides with $J_{\text {Id,Id }}^{(1)}$ from eq. (3.2) upon setting $s:=-\alpha^{\prime} s_{12}$ and $t:=-\alpha^{\prime} s_{13}$. The integral defining $V(s, t)$ is absolutely convergent for $s, t \in \mathbb{C}$ such that $\operatorname{Re}(s)>0, \operatorname{Re}(t)>0$ and $\operatorname{Re}(s+t)<1$.

The integrand is entire in $s$ and $t$ as soon as $z$ belongs to the domain $D_{\varepsilon}$ obtained from $\mathbb{P}_{\mathbb{C}}^{1}$ by cutting out small discs of radius $\varepsilon$ around $z=0,1, \infty$, where it can therefore be expanded as a power series at $(s, t)=(0,0)$. Hence we can write

$$
V(s, t)=\lim _{\varepsilon \rightarrow 0} \int_{D_{\varepsilon}} \sum_{p, q \geq 0} s^{p} t^{q} \frac{\mathcal{L}_{x_{0}^{p}}(z) \mathcal{L}_{x_{1}^{q}}(z)}{|z|^{2}|1-z|^{2}} d^{2} z,
$$

where we recall that $\mathcal{L}_{x_{0}^{p}}(z)=\left(\log |z|^{2}\right)^{p} / p$ ! and $\mathcal{L}_{x_{1}^{q}}(z)=\left(\log |1-z|^{2}\right)^{q} / q$ !. Exchanging integration 
with summation and rearraging the summation ${ }^{3}$, one finds

$$
V(s, t)=\lim _{\varepsilon \rightarrow 0}\left(\sum_{p, q \geq 1} s^{p} t^{q} \int_{D_{\varepsilon}} \frac{\mathcal{L}_{x_{0}^{p}}(z) \mathcal{L}_{x_{1}^{q}}(z)}{|z|^{2}|1-z|^{2}} d^{2} z+\sum_{\substack{p, q \geq 0 \\ p \cdot q=0}} s^{p} t^{q} \int_{D_{\varepsilon}} \frac{\mathcal{L}_{x_{0}^{p}}(z) \mathcal{L}_{x_{1}^{q}}(z)}{|z|^{2}|1-z|^{2}} d^{2} z\right) .
$$

We focus now on the first term. Because the map $L_{w}(z) \rightarrow \mathcal{L}_{w}(z)$ respects shuffle product identities and the $\mathrm{KZ}$ equation, we get

$$
\frac{\mathcal{L}_{x_{0}^{p}}(z) \mathcal{L}_{x_{1}^{q}}(z)}{|z|^{2}|1-z|^{2}}=\frac{\partial}{\partial z}\left(\sum_{w=x_{0}^{p} \amalg x_{1}^{q}} \frac{\mathcal{L}_{x_{0} w}(z)-\mathcal{L}_{x_{1} w}(z)}{\bar{z}(1-\bar{z})}\right) .
$$

Using this fact, the Stokes theorem and the known asymptotic behaviour of multiple polylogarithms at $0,1, \infty$, and denoting by $B_{z}(\varepsilon)$ a disc centered at $z$ of radius $\varepsilon$, one finds that for any $p, q \geq 1$

$$
\begin{aligned}
\int_{D_{\varepsilon}} \frac{\mathcal{L}_{x_{0}^{p}}(z) \mathcal{L}_{x_{1}^{q}}(z)}{|z|^{2}|1-z|^{2}} d^{2} z & =-\frac{1}{2 \pi i} \sum_{z=0,1, \infty}\left(\int_{\bar{\partial} B_{z}(\varepsilon)} \frac{d \bar{z}}{\bar{z}(1-\bar{z})} \sum_{w=x_{0}^{p} \amalg x_{1}^{q}}\left(\mathcal{L}_{x_{0} w}(z)-\mathcal{L}_{x_{1} w}(z)\right)\right) \\
& =\sum_{w=x_{0}^{p} \uplus x_{1}^{q}}\left(\mathcal{L}_{x_{0} w}(1)-\mathcal{L}_{x_{1} w}(1)\right)+O(\varepsilon) .
\end{aligned}
$$

This method to compute integrals of single-valued multiple polylogarithms is sometimes referred to as single-valued integration and was introduced by Schnetz in [58] (see also [59] for an application to multi-Regge kinematics). By a more careful analysis of the dependence on $\varepsilon$ one concludes that

$$
\sum_{p, q \geq 1} s^{p} t^{q} \int_{D_{\varepsilon}} \frac{\mathcal{L}_{x_{0}^{p}}(z) \mathcal{L}_{x_{1}^{q}}(z)}{|z|^{2}|1-z|^{2}} d^{2} z=\sum_{p, q \geq 1}\left(\sum_{w=x_{0}^{p} \amalg x_{1}^{q}} \mathcal{L}_{x_{0} w}(1)-\mathcal{L}_{x_{1} w}(1)\right) s^{p} t^{q}+O(\varepsilon) .
$$

The second term of (3.14) is treated similarly. We need to separate the contributions given by the Stokes theorem applied on the boundaries of the three discs $B_{0}(\varepsilon), B_{1}(\varepsilon), B_{\infty}(\varepsilon)$. It turns out ${ }^{3}$ that the contribution from $B_{0}(\varepsilon)$ is

$$
-\sum_{p \geq 0}\left(\frac{\left(\log \varepsilon^{2}\right)^{p+1}}{(p+1) !}\right) s^{p}+O(\varepsilon)=\frac{1}{s}\left(1-\varepsilon^{2 s}\right)+O(\varepsilon),
$$

the contribution from $B_{\infty}(\varepsilon)$ is $O(\varepsilon)$ and the contribution from $B_{1}(\varepsilon)$ is

$$
\begin{aligned}
& \sum_{q \geq 1} \mathcal{L}_{x_{0} x_{1}^{q}}(1) t^{q}-\sum_{q \geq 0}\left(\frac{\left(\log \varepsilon^{2}\right)^{q+1}}{(q+1) !}\right) t^{q}-\sum_{p \geq 1} \mathcal{L}_{x_{1} x_{0}^{p}}(1) s^{p}+O(\varepsilon) \\
& =\sum_{q \geq 1} \mathcal{L}_{x_{0} x_{1}^{q}}(1) t^{q}+\frac{1}{t}\left(1-\varepsilon^{2 t}\right)-\sum_{p \geq 1} \mathcal{L}_{x_{1} x_{0}^{p}}(1) s^{p}+O(\varepsilon) .
\end{aligned}
$$

${ }^{3}$ We refer to [38] for a justification of these steps and details. 
Taking the limit $\varepsilon \rightarrow 0$ and noting that $\mathcal{L}_{x_{0} x_{1}^{n}}(1)=-\mathcal{L}_{x_{1} x_{0}^{n}}(1)=\zeta^{\mathrm{sv}}(n+1)$ for $n \geq 1$, we obtain

$$
V(s, t)=\frac{s+t}{s t}+2 \sum_{n \geq 1} \zeta(2 n+1)\left(s^{2 n}+t^{2 n}\right)+\sum_{p, q \geq 1}\left(\sum_{w=x_{0}^{p} \amalg x_{1}^{q}} \mathcal{L}_{x_{0} w}(1)-\mathcal{L}_{x_{1} w}(1)\right) s^{p} t^{q}
$$

We deduce that $V(s, t)$ can be analytically continued to define a meromorphic function in a neighborhood of $(s, t)=(0,0)$, and the coefficients of its Laurent series expansion at that point belong to the ring $\mathcal{Z}^{\text {sv }}$ of single-valued multiple zeta values.

Of course, this result was already clear from eq. (2.30), which implies the stronger fact that the coefficients of $V(s, t)$ are polynomials in odd zeta values. The advantage of the computation above is that it can be generalized to higher-point amplitudes. To do this, the idea is to apply the single-valued integration recursively, but it is necessary to overcome two difficulties. The first is that the singularity divisors coming from the integrand are not normal crossing, and this can be solved with standard geometric or analytic methods $[38,50]$. The second is that it is necessary to work with a more general set of functions called single-valued hyperlogarithms, defined by Brown in [78], which are analogues of single-valued multiple polylogarithms arising from more general alphabets $X$, whose letters correspond to punctures on the Riemann sphere. The successive integration of these functions requires to recursively remove the integration variables from the alphabet. Methods to solve this kind of problem were developed in [38] (precisely to prove that the coefficients of closed string tree-level amplitudes are single-valued multiple zeta values) or, with a different approach relying on standard conjectures on multiple zeta values, in [59].

\section{Part III}

\section{One-loop amplitudes}

\section{Closed string building blocks and modular graph functions}

At one-loop, closed oriented string amplitudes are defined on the complex torus, and can be decomposed on building blocks as [18, 67, 68]

$$
M_{N+1}(\boldsymbol{s}, \boldsymbol{\epsilon})=\sum_{r} c_{r}^{1-\text { loop }}(\boldsymbol{s}, \boldsymbol{\epsilon}) M_{N+1}^{1-\text { loop }}\left(\boldsymbol{s}, \boldsymbol{n}^{r}, \tilde{\boldsymbol{n}}^{r}\right)
$$

with the one-loop building blocks given by the integrals over the moduli space of genus-one closed Riemann surfaces ${ }^{4}$ (the complex tori) with $N+1$ marked points $\mathfrak{M}_{1, N+1}$. This integration domain can

\footnotetext{
${ }^{4}$ Bosonic strings are not consistent at loop order because of the tachyons in the spectrum. For superstring theory amplitudes, at higher genus the projection of the supermoduli integral onto the bosonic moduli space of genus $g$ curves with marked point cannot be done using a naive integration of the fermonic variables [79, 80], and may be achieved using Sen's fermionic integration [81, 82].
} 
be factorized, so that the building blocks take the form

$$
M_{N+1}^{1-\text { loop }}(\boldsymbol{s}, \boldsymbol{n}, \tilde{\boldsymbol{n}})=\int_{\mathfrak{M}_{1,1}} \frac{d \tau_{1} d \tau_{2}}{\tau_{2}^{2}} \hat{M}_{N+1}^{1-\mathrm{loop}}(\boldsymbol{s}, \boldsymbol{n}, \tilde{\boldsymbol{n}} ; \tau)
$$

with the partial (configuration-space) amplitude building blocks

$$
\begin{aligned}
& \hat{M}_{N+1}^{1-\text { loop }}(\boldsymbol{s}, \boldsymbol{n}, \tilde{\boldsymbol{n}} ; \tau)= \\
& \quad \int_{\mathbb{T}^{N}} \prod_{i=1}^{N} \frac{i d z_{i} d \bar{z}_{i}}{2 \tau_{2}} \prod_{1 \leq i<j \leq N+1} e^{2 \alpha^{\prime} s_{i j} G^{\mathbb{T}}\left(z_{i}, z_{j} ; \tau\right)}\left(\partial_{z_{i}} G^{\mathbb{T}}\left(z_{i}, z_{j} ; \tau\right)\right)^{-2 n_{i j}}\left(\partial_{\bar{z}_{i}} G^{\mathbb{T}}\left(z_{i}, z_{j} ; \tau\right)\right)^{-2 \tilde{n}_{i j}},
\end{aligned}
$$

where we fix $z_{N+1}:=0$. The exponents $n_{i j}$ and $\tilde{n}_{i j}$ are non-positive integers arising from the operator product expansion. The Arakelov scalar Green function $G^{\mathbb{T}}\left(z, z^{\prime} ; \tau\right)$ on the complex torus $\mathbb{T}:=\mathbb{C} /(\mathbb{Z}+\tau \mathbb{Z})$ of modulus $\tau=\tau_{1}+i \tau_{2} \in \mathbb{H}$ is defined by

$$
\partial_{z} \partial_{\bar{z}} G^{\mathbb{T}}\left(z, z^{\prime} ; \tau\right)=-\frac{\pi}{2} \delta^{(2)}\left(z-z^{\prime}\right)+\frac{\pi}{2 \tau_{2}}, \quad \quad \int_{\mathbb{T}} G^{\mathbb{T}}(z, 0 ; \tau) d z d \bar{z}=0 .
$$

This determines the Green function to be given by

$$
G^{\mathbb{T}}\left(z, z^{\prime} ; \tau\right)=-\frac{1}{2} \log \left|\frac{\theta_{1}\left(z-z^{\prime}, \tau\right)}{\eta(\tau)}\right|^{2}+\frac{\pi \mathfrak{I m}\left(z-z^{\prime}\right)^{2}}{\tau_{2}},
$$

with the ratio of the odd Jacobi $\theta_{1}$-function and the Dedekind $\eta$-function given, setting $q=e^{2 \pi i \tau}$, by

$$
\frac{\theta_{1}(z, \tau)}{\eta(\tau)}=i q^{\frac{1}{12}} e^{-i \pi z}\left(1-e^{2 \pi i z}\right) \prod_{n \geq 1}\left(1-q^{n} e^{2 i \pi z}\right)\left(1-q^{n} e^{-2 i \pi z}\right) .
$$

We have used the Bern-Kosower representation of one-loop amplitudes with only first derivatives of the Green function after using integration by parts in the integrand [83]. This decomposition is the one-loop equivalent of the decomposition of tree-level amplitudes in (1.1) on the building blocks (1.4).

In this paper we will focus on the partial amplitude building blocks in (4.3), rather than the moduli space integrals giving the full amplitude building blocks in (4.2). The coefficients of the small $\alpha^{\prime}$ expansion of the partial amplitudes building blocks are given by modular graph functions [60,84] and modular graph forms [62], which constitute interesting new classes of real-analytic modular functions and forms, respectively. For the purposes of this paper, it is sufficient to focus on modular graph functions, which is the topic of the next sections. In particular, in section 4.2 we present a new application of the single-valued integration from section 3.2 to compute their asymptotic expansion in the two-point case. 



Figure 4.1: Examples of graphs associated to modular graph functions

\subsection{Modular graph functions}

We consider a graph ${ }^{5} \Gamma$ drawn on a fundamental domain $\Sigma_{\tau} \subset \mathbb{C}$ of the torus. Let $z_{i}$ with $i=1, \ldots, N+1$ be the vertices of the graph. By translation invariance, we can suppose that $0 \in \Sigma_{\tau}$ and fix $z_{N+1}=0$. We denote by $e_{i j} \geq 0$ the number of edges connecting the points $i$ and $j$. The modular graph functions [60] are defined by the following integrals associated to the graph $\Gamma$ :

$$
D_{\Gamma}(\tau)=\int_{\Sigma_{\tau}^{N}} \prod_{1 \leq i<j \leq N+1}\left(G^{\mathbb{T}}\left(z_{i}, z_{j} ; \tau\right)\right)^{e_{i j}} \prod_{i=1}^{N} \frac{i d z_{i} d \bar{z}_{i}}{2 \tau_{2}} .
$$

The word modular refers to the easily checked fact that modular graph functions are invariant under the standard action of $\mathrm{SL}_{2}(\mathbb{Z})$ on $\tau \in \mathbb{H}$. These functions arise naturally in the $\alpha^{\prime}$-expansion of the partial amplitude building blocks in (4.3) with $n_{i j}=\tilde{n}_{i j}=0$. When $n_{i j}$ and $\tilde{n}_{i j}$ are non-vanishing, one gets modular graph forms [62], which have non-trivial holomorphic and anti-holomorphic modular weights with respect to the action of $\mathrm{SL}_{2}(\mathbb{Z})$.

It was proven in [85] that, if we denote by $E$ the total number of edges of $\Gamma$, the asymptotic expansion as $\tau \rightarrow i \infty$ of modular graph functions takes the form

$$
D_{\Gamma}(\tau)=\sum_{k=1-E}^{E} \sum_{m, n \geq 0} d_{k}^{(m, n)}(\Gamma) Y^{k} q^{m} \bar{q}^{n}
$$

where $Y:=\pi \tau_{2}$ and $d_{k}^{(m, n)}(\Gamma)$ belong to the ring generated by all special values at roots of unity of multiple polylogarithms. A refinement of this theorem was recently announced by E. Panzer [86], who proved that $d_{k}^{(m, n)}(\Gamma)$ belong to the ring $\mathcal{Z}$ of multiple zeta values. Conjecturally, however, this is still not the optimal result, as the coefficients $d_{k}^{(m, n)}(\Gamma)$ are expected to belong to the smaller ring $\mathcal{Z}^{\mathrm{sv}}$ of single-valued multiple zeta values $[60,87]$. It is presumably straightforward to see that all this applies as well to modular graph forms.

The first Laurent polynomial in this expansion, obtained setting $m=n=0$, is usually called the "constant term". Its knowledge is particularly relevant because it can be used to deduce algebraic and differential relations among modular graph functions (and forms) [62, 88-91], but its computation is extremely hard for graphs with more than two loops or two points [87, 92]. From the geometric viewpoint, it is the main term as $\tau \rightarrow i \infty$, which is the degeneration limit of the complex structure of

\footnotetext{
${ }^{5}$ More precisely, we consider an undirected graph with multiple edges connecting pairs of vertices and no self-edges.
} 
the torus towards a sphere with two extra marked points. For this reason, it is expected to be related to tree-level amplitudes on a sphere, and this would explain the conjectural relation to single-valued multiple zeta values. We will see in the next section that these expectations can be verified in the simple case of two-point graphs.

\subsection{The banana graphs}

We look here at the constant term of the asymptotic expansion in the case of two-point modular "banana graph" functions $D_{n}(\tau)=$ of the simplest families of graphs. It was shown in [36,63] that the coefficients $d_{k}^{(0,0)}$ of the constant term can be written in terms of the coefficients of the four-point closed string amplitude in eq. (1.5), which belong to the ring $\mathcal{Z}^{\mathrm{sv}}$ as shown in section 3.2 and which are actually contained in the smaller. subring generated by odd zeta values, as diplayed in eq. (2.30).

We give here an alternative proof that the coefficients $d_{k}^{(0,0)}$ are single-valued multiple zeta values exploiting the single-valued integration already used in section 3.2. This proof is inspired by the approach of [63].

A generating series for the two-point functions $D_{n}(\tau)$ is

$$
F(s, \tau):=\sum_{n \geq 0} \frac{s^{n}}{n !} D_{n}(\tau)=\int_{\Sigma_{\tau}} e^{s G^{\mathbb{T}}(z, 0 ; \tau)} \frac{i d z d \bar{z}}{2 \tau_{2}}
$$

where $G^{\mathbb{T}}\left(z, z^{\prime} ; \tau\right)$ is the genus one closed string Green function defined in (4.5). The integral on the right hand side coincides with the two-point closed string building block $\hat{M}_{2}^{1-\text { loop }}(\boldsymbol{s}, \mathbf{0}, \mathbf{0} ; \tau)$ upon setting $s:=2 \alpha^{\prime} s_{12}$.

As in the previous section, we set $Y:=\pi \tau_{2}$. Moreover, we denote $\alpha:=\operatorname{Re}(z)$ and $\beta:=\operatorname{Jm}(z) / \tau_{2}$ so that the standard measure $\frac{i d z d \bar{z}}{2 \tau_{2}}$ on $\Sigma_{\tau}$ can be written as $d \alpha d \beta$. Finally, we fix the fundamental domain $\alpha \in[0,1], \beta \in\left[-\frac{1}{2}, \frac{1}{2}\right]$. In this domain, all contributions to the integral coming from the infinite product $\prod_{n \geq 1} \cdots$ in equation (4.6) can be ignored for large $Y$, and we find

$$
F(s, \tau)=\int_{0}^{1} e^{2 Y s\left(\beta^{2}-\beta+\frac{1}{6}\right)} d \beta+e^{s Y / 3} \int_{-\frac{1}{2}}^{\frac{1}{2}} \int_{0}^{1} e^{2 Y s \beta^{2}} e^{-2 Y s \beta}\left(\left|1-e^{2 \pi i \alpha-2 Y \beta}\right|^{-2 s}-1\right) d \alpha d \beta+O\left(e^{-2 Y}\right) .
$$

The first integral gives the leading terms of the Laurent polynomials (see [84, §D.1]):

$$
\int_{0}^{1} e^{2 Y s\left(\beta^{2}-\beta+\frac{1}{6}\right)} d \beta=\sum_{n \geq 0} \frac{(Y s)^{n}}{3^{n} n !}{ }_{2} F_{1}\left(\begin{array}{c}
1,-n \\
\frac{3}{2}
\end{array} \mid \frac{3}{2}\right),
$$

where ${ }_{2} F_{1}\left(\begin{array}{c}a b \\ c\end{array} \mid z\right)=\sum_{n \geq 0} \frac{\Gamma(a+n) \Gamma(b+n) \Gamma(c)}{\Gamma(a) \Gamma(b) \Gamma(c+n)} \frac{z^{n}}{n !}$ is the Gauss hypergeometric function.

In the second integral, we make the change of variables $\zeta=\exp (2 \pi i \alpha-2 Y \beta)$, we Taylor expand 
the integrand and we exchange integration with summation (by holomorphicity at $s=0$ ) to obtain

$$
\frac{e^{s Y / 3}}{4 Y} \sum_{\substack{k, n \geq 0 \\ m \geq 1}} \frac{(2 k+n) !}{k ! n !} \frac{(-1)^{m}}{2^{n+3 k}} \frac{s^{k+m+n}}{Y^{k}} \int_{|q|^{\frac{1}{2}} \leq|\zeta| \leq|q|^{-\frac{1}{2}}} \frac{\mathcal{L}_{x_{0}^{2 k+n}}(\zeta) \mathcal{L}_{x_{1}^{m}}(\zeta)}{|\zeta|^{2}} d^{2} \zeta+O\left(e^{-2 Y}\right)
$$

where we employ the notation $\mathcal{L}_{x_{0}^{r}}(\zeta)=(\log |\zeta|)^{r} / r$ ! and $\mathcal{L}_{x_{1}^{r}}(\zeta)=(\log |1-\zeta|)^{r} / r$ ! from section 3.1 and we denote $d^{2} \zeta:=i d \zeta d \bar{\zeta} / 2 \pi$.

Because the map $L_{w}(z) \rightarrow \mathcal{L}_{w}(z)$ respects shuffle product identities and the KZ equation, we get $\Omega$

$$
\frac{\mathcal{L}_{x_{0}^{2 k+n}}(\zeta) \mathcal{L}_{x_{1}^{m}}(\zeta)}{|\zeta|^{2}} d^{2} \zeta=\sum_{w \in x_{0}^{2 k+n} \amalg x_{1}^{m}} \frac{\mathcal{L}_{w}(\zeta)}{|\zeta|^{2}} d^{2} \zeta=d\left(\sum_{w \in 0^{2 k+n} \amalg 1^{m}} \frac{\mathcal{L}_{0 w}(\zeta)}{\bar{\zeta}} \frac{i d \bar{\zeta}}{2 \pi}\right)
$$

hence by applying the Stokes theorem we find

$$
\begin{aligned}
& \int_{|q|^{\frac{1}{2}} \leq|\zeta| \leq|q|^{-\frac{1}{2}}} \frac{\mathcal{L}_{x_{0}^{2 k+n}}(\zeta) \mathcal{L}_{x_{1}^{m}}(\zeta)}{|\zeta|^{2}} d^{2} \zeta= \\
& =\int_{0}^{2 \pi} \sum_{w \in x_{0}^{2 k+n} \amalg x_{1}^{m}} \mathcal{L}_{x_{0} w}\left(|q|^{-\frac{1}{2}} e^{i \theta}\right) \frac{d \theta}{2 \pi}-\int_{0}^{2 \pi} \sum_{w \in x_{0}^{2 k+n} \amalg x_{1}^{m}} \mathcal{L}_{x_{0} w}\left(|q|^{\frac{1}{2}} e^{i \theta}\right) \frac{d \theta}{2 \pi} .
\end{aligned}
$$

Integrating term-by-term the asymptotic expansion of single-valued multiple polylogarithms at 0 , which can be easily deduced from (3.5), one finds that the second integral on the right hand side is $O\left(e^{-2 Y}\right)$. As for the first integral, since we are now interested in large $Y=\log |q|^{-1 / 2}$, we need to know the asymptotic behaviour of single-valued multiple polylogarithms at $\infty$. By Lemma 2.17 of [58] we have the generating series identity

$$
\mathcal{L}_{\left\{x_{0}, x_{1}\right\}}\left(z^{-1}\right)=\mathcal{L}_{\left\{-x_{0}-x_{1}, x_{1}\right\}}(z) \mathcal{L}_{\left\{x_{0},-x_{0}-x_{1}\right\}}(1)
$$

which together with the asymptotic behaviour at 0 implies that there exists an integer $K_{\infty} \geq 0$ (depending on $w$ ) such that in a neighbourhood of $\infty$

$$
\mathcal{L}_{w}(z)=\sum_{i, j \geq 0} \sum_{k=0}^{K_{\infty}} c_{i, j, k}^{(\infty)} \frac{\left(\log |z|^{2}\right)^{k}}{z^{i} \bar{z}^{j}}
$$

with $c_{i, j, k}^{(\infty)} \in \mathcal{Z}^{\mathrm{sv}}$. Combining this with (4.14) implies that there exist an integer $R(k, m, n) \geq 0$ and numbers $Z_{r}(k, m, n) \in \mathcal{Z}^{\mathrm{sv}}$ such that

$$
\int_{|q|^{\frac{1}{2}} \leq|\zeta| \leq|q|^{-\frac{1}{2}}} \frac{\mathcal{L}_{x_{0}^{2 k+n}}(\zeta) \mathcal{L}_{x_{1}^{m}}(\zeta)}{|\zeta|^{2}} d^{2} \zeta=\sum_{r=0}^{R(k, m, n)} Z_{r}(k, m, n)(2 Y)^{r}+O\left(e^{-2 Y}\right)
$$


We conclude that for large $Y$ we have

$$
\begin{aligned}
F(s, \tau) & =\sum_{n \geq 0} \frac{(Y s)^{n}}{3^{n} n !}{ }_{2} F_{1}\left(\begin{array}{c}
1,-n \\
\frac{3}{2}
\end{array} \mid \frac{3}{2}\right) \\
& +\frac{e^{s Y / 3}}{4 Y} \sum_{\substack{k, n \geq 0 \\
m \geq 1}} \frac{(2 k+n) !}{k ! n !} \frac{(-1)^{m}}{2^{n+3 k}} \frac{s^{k+m+n}}{Y^{k}} \sum_{r=0}^{R(k, m, n)} Z_{r}(k, m, n)(2 Y)^{r}+O\left(e^{-2 Y}\right) .
\end{aligned}
$$

This proves that the coefficients of the constant term in the asymptotic expansion of the two-point graph functions $D_{n}(\tau)$ belong to the ring of single-valued multiple zeta values. Even though this proof does not explicitly relate these numbers with the coefficients of a genus-zero amplitude, and does not imply the stronger statement that they belong to the subring generated by odd Riemann zeta values, we believe that it may be of interest because of its potential to generalize to a higher number of points, thanks to the generality of the single-valued integration developed in [38].

\section{Open string building blocks and open questions}

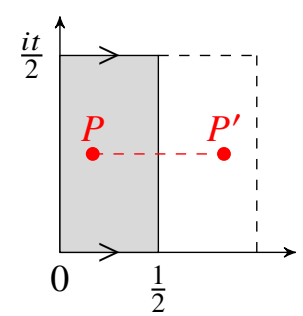

(a)



(b)

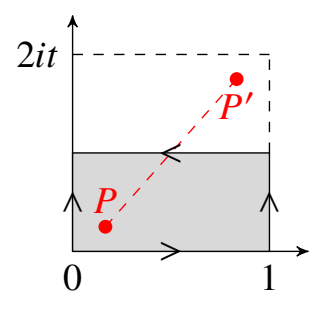

(c)

Figure 5.1: Grey area represents the fundamental cell of (a) the annulus $\mathcal{A}$, (b) the Möbius strip $\mathcal{M}$ and (c) the Klein bottle $\mathcal{K}$. The points $P$ and $P^{\prime}$ are identified under the action of the involution on the covering torus. For the annulus and Möbius strip the involution is $\mathcal{I}_{\mathcal{A}}(z)=\mathcal{I}_{\mathcal{M}}(z)=1-\bar{z}$ and for the Klein bottle $\mathcal{I}_{\mathcal{K}}(z)=1-\bar{z}+\frac{\tau_{\mathcal{K}}}{2}$.

The remaining one-loop topologies are the annulus (open oriented sector), the Möbius strip (open unoriented sector) and the Klein bottle (closed unoriented sector). The associated amplitudes can be written in terms of building blocks given by integrals on the moduli spaces of annuli, Möbius strips and Klein bottles [93, 94]. Similarly to the torus case, one can factorise the integration domains of the building blocks and obtain partial amplitude building blocks given by integrals over configuration spaces of points on the relevant surface. The form of these integrals strongly depends on the topology of the worldsheet. We start by reviewing the involutions on the covering torus and the Green functions obtained with the method of images, following [22].

- The annulus $\mathcal{A}$ is obtained by the action of the involution $\mathcal{I}_{\mathcal{A}}(z)=1-\bar{z}$ on the doubly covering torus of modulus $\tau_{\mathcal{A}}=\frac{i t}{2}$ with $t \in \mathbb{R}^{+}$, as represented in figure 5.1(a). The two boundaries of the annulus are at $\operatorname{Re}(z)=0$ and $\operatorname{Re}(z)=\frac{1}{2}$, and a fundamental cell is given by $\left[0, \frac{1}{2}\right] \times\left[0, \frac{t}{2}\right]$. The method of images applied to the torus propagator in (4.5), together with well-known properties of $\theta_{1}$, gives

$$
G^{\mathcal{A}}\left(z, z^{\prime} ; \tau_{\mathcal{A}}\right)=G^{\mathbb{T}}\left(z, z^{\prime} ; \tau_{\mathcal{A}}\right)+G^{\mathbb{T}}\left(z, 1-\bar{z}^{\prime} ; \tau_{\mathcal{A}}\right)
$$


- The Möbius strip is obtained by the action of the involution $\mathcal{I}_{\mathcal{M}}(z)=1-\bar{z}$ on the doubly covering torus of modulus $\tau_{\mathcal{M}}=\frac{1}{2}+i \frac{t}{2}$ with $t \in \mathbb{R}^{+}$, as represented in figure 5.1(b). The boundary of the Möbius strip is located at $\mathfrak{R e}(z)=\frac{1}{2}$ and $\mathfrak{R e}(z)=1$, and a fundamental cell is given by $\left[\frac{1}{2}, 1\right] \times\left[0, \frac{t}{2}\right]$. Similarly to the annulus case, the method of images applied to the torus propagator in (4.5) gives

$$
G^{\mathcal{M}}\left(z, z^{\prime} ; \tau_{\mathcal{M}}\right)=G^{\mathbb{T}}\left(z, z^{\prime} ; \tau_{\mathcal{M}}\right)+G^{\mathbb{T}}\left(z, 1-\bar{z}^{\prime} ; \tau_{\mathcal{M}}\right)=G^{\mathcal{A}}\left(z, z^{\prime} ; \tau_{\mathcal{M}}\right)
$$

- The Klein bottle $\mathcal{K}$ is obtained by the action of the involution $\mathcal{I}_{\mathcal{K}}(z)=1-\bar{z}+\frac{\tau_{\mathcal{K}}}{2}$ on the doubly covering torus of modulus $\tau_{\mathcal{K}}=2 i t$ with $t \in \mathbb{R}^{+}$, as represented in figure 5.1(c). There are no boundary components, and a fundamental cell is given by $[0,1] \times[0, t]$. The method of images applied to the torus propagator in (4.5) this time gives

$$
G^{\mathcal{K}}\left(z, z^{\prime} ; \tau_{\mathcal{K}}\right)=G^{\mathbb{T}}\left(z, z^{\prime} ; \tau_{\mathcal{K}}\right)+G^{\mathbb{T}}\left(z, 1-\bar{z}+\frac{\tau_{\mathcal{K}}}{2} ; \tau_{\mathcal{K}}\right)
$$

These similar expressions for the Green functions suggest that the respective (partial ${ }^{6}$ ) amplitudes could be related to each others. It was argued in various cases that, indeed, one can reduce Möbius strip and Klein bottle partial amplitudes to annulus partial amplitudes [24, 95]. As we will discuss in section 5.2, however, very little is known about these amplitudes in all cases which involve closed string states.

The only case which was systematically studied is that of open string partial amplitude building blocks given by ordered integrals on the boundaries of the annulus or of the Möbius strip. We report in the next section some recent observation about their relation to the closed string building blocks (4.3) via the same single-valued projection mentioned at tree-level.

\subsection{Open string partial amplitudes on the annulus}

One-loop open string partial amplitudes are given by ordered integrals on the boundaries of Möbius strips and annuli. The annulus case can be divided into the planar case, with string states only on one boundary, and the non-planar case involving both boundaries. The Möbius strip case can be reduced to the planar annulus case, as explained for instance in [66]. Moreover, as remarked in [32, 35, 66], the non-planar annulus case seems to share all the mathematical features of the planar case. For this reason, we only focus on partial amplitude building blocks with all insertions on one boundary of the annulus. We fix these building blocks ${ }^{7}$ to be given by the following iterated integrals over the straight path $[0, \tau]$ with $\tau=\tau_{\mathcal{A}}=i t / 2 \in i \mathbb{R}^{+}$(or any other fundamental domain of the annulus boundary $\tau \mathbb{R} / \tau \mathbb{Z}$ depicted in figure 5.1(a)):

\footnotetext{
${ }^{6} \mathrm{As}$ in the torus case, we are mostly interested in the partial amplitudes, given by integrals over configuration spaces.

${ }^{7}$ We integrate over the B-cycle of the torus, instead of the A-cycle used in most recent references, to be consistent with our choice of involution given in figure 5.1(a).
} 


$$
\hat{A}_{N+1}^{1-\text { loop }}(\boldsymbol{s}, \boldsymbol{n}, \rho ; \tau)=\int_{0 \leq z_{\rho(1)} \leq \cdots \leq z_{\rho(N)} \leq \tau} \prod_{i=1}^{N} \frac{d z_{i}}{\tau_{2}} \prod_{1 \leq i<j \leq N+1} e^{2 \alpha^{\prime} s_{i j} G^{\mathcal{A}}\left(z_{i}, z_{j} ; \tau\right)}\left(\partial_{z_{i}} G^{\mathcal{A}}\left(z_{i}, z_{j} ; \tau\right)\right)^{-2 n_{i j}}
$$

where we set $z_{N+1}:=0$. The Green function $G^{\mathcal{A}}\left(z, z^{\prime} ; \tau\right)$ from (5.1) reduces for $z, z^{\prime} \in \tau \mathbb{R} / \tau \mathbb{Z}$ and $\tau \in i \mathbb{R}^{+}$to

$$
G^{\mathcal{A}}\left(z, z^{\prime} ; \tau\right)=-2 \log \left|\frac{\theta_{1}\left(z-z^{\prime}, \tau\right)}{\eta(\tau)}\right|-\frac{2 \pi i\left(z-z^{\prime}\right)^{2}}{\tau} .
$$

It was shown in [64] that the coefficients of the small $\alpha^{\prime}$ expansion of the integrals (5.4) (or rather their analytic continuation for $\tau \in \mathbb{H}$ ) can be written in terms of elliptic multiple zeta values. The latter are functions on the complex upper half-plane which constitute the natural genus-one generalization of multiple zeta values and arise from the genus-one Knizhnik-Zamolodchikov-Bernard connection [96]. In particular, in the limit $\tau \rightarrow i \infty$ one lands on the space of classical multiple zeta values. This is consistent with the fact that the limit of the integrals (5.4) can be expressed in terms of $(N+3)$-point tree-level building blocks [34].

From the cohomological viewpoint, elliptic multiple zeta values are periods of the configuration space of points on the torus, and it is conjectured that modular graph functions and forms belong to the family constituting their single-valued analogues [97]. This would be consistent with the genus-zero situation, and also with the conjecture that the coefficients of the constant terms of modular graph functions are single-valued multiple zeta values. Some evidence of an extension of the genus-zero single-valued projection between open and closed string building blocks was found in [32, 33, 86, 98]. In the next section we report on one of the conjectures related to this single-valued projection, which was recently proven in the two-point case [36].

\subsubsection{The two-point case}

We consider the open string integral

$$
\int_{z \in[0, \tau]} e^{s \hat{G}^{\mathcal{P}}(z, 0 ; \tau)} \frac{d z}{\tau_{2}}
$$

This integral coincides with the building block $\hat{A}_{2}^{1-\text { loop }}(s, 0, \mathrm{Id} ; \tau)$ upon setting $s:=2 \alpha^{\prime} s_{12}$ and replacing the Green function $G^{\mathcal{A}}\left(z, z^{\prime} ; \tau\right)$ from (5.5) with the modified version introduced in [32]

$$
\hat{G}^{\mathcal{A}}\left(z, z^{\prime} ; \tau\right):=G^{\mathcal{A}}\left(z, z^{\prime} ; \tau\right)-\frac{\pi i}{3 \tau},
$$

which satisfies the condition $\int_{[0, \tau]} \hat{G}^{\mathcal{A}}(z, 0 ; \tau) d z=0$. The correction to the Green function is constant in $z$, so it disappears by momentum conservation when we consider the two-point amplitude (which is not physically meaningful) as contributing to higher-point amplitudes.

The integral in (5.6) defines a holomorphic function of $s$ at the origin, whose $n$-th Taylor coefficient 
at $s=0$ (i.e. in the limit $\alpha^{\prime} \rightarrow 0$ ) is given up to a constant factor by the integral

$$
B_{n}(\tau):=\frac{1}{4^{n}} \int_{z \in[0, \tau]} \hat{G}^{\mathcal{A}}(z, 0 ; \tau)^{n} \frac{d z}{\tau_{2}}
$$

which can be analytically continued to define a holomorphic function of $\tau \in \mathbb{H}$. These Taylor coefficients are special cases of a class of functions arising from symmetrized open string integrals, called holomorphic graph functions, which constitute holomorphic analogues of modular graph functions [32,99]. More precisely, the functions $B_{n}(\tau)$ are exactly those holomorphic graph functions associated to the two-points banana graphs, and so they are the open-string analogues of the two-point modular graph functions $D_{n}(\tau)$ from section 4.2.

It was proven in [99] that all holomorphic graph functions (associated to a graph $\Gamma$ with $E$ edges) have the asymptotic expansion

$$
\sum_{k=-E}^{E} \sum_{m \geq 0} b_{k}^{(m)}(\Gamma) T^{k} q^{m},
$$

where $T:=\pi \tau / 2 i$ and $b_{k}^{(m)}$ belong to the ring $\mathcal{Z}$ of multiple zeta values. This expansion is similar to the asymptotic expansion (4.8) of modular graph functions, and it was conjectured in [32] that the coefficients $b_{k}^{(0)}(\Gamma)$ of the leading Laurent polynomial are mapped precisely to the corresponding coefficients $d_{k}^{(0,0)}(\Gamma)$ via the single-valued projection on multiple zeta values.

This conjecture was recently proven for the two-point banana graphs in [36]. In other words, one can map the asymptotic limit of the two-point open string integral (5.6) to the asymptotic limit of the two-point closed string integral (4.9) using the single-valued projection on multiple zeta values. Moreover, these asymptotic limits were also shown to be related by some double-copy formula [36]. This is not so surprising, if we think that the tree-level KLT double-copy relations can be seen as part of the same cohomological framework which explains also the single-valued projection [50]. For this reason, relating the whole asymptotic expansions via the theory of single-valued periods would probably also point towards a first genus-one analogue of the KLT relations.

\subsection{Open questions}

We have already mentioned that it is not clear in general how the building blocks of annulus, Möbius strip and Klein bottle partial amplitudes relate to each others. It would be interesting to know, for instance, whether we can reduce them to the open string building blocks (5.4) and thus obtain the same kind of structure which occurs at tree-level. One could also ask the (less ambitious) question of what is the minimal set of special functions which appear in the $\alpha^{\prime}$-expansion of these partial amplitudes: are elliptic multiple zeta values sufficient?

Moreover, at tree-level we have a clear picture not only of the building blocks, but also of the relations among them, namely the KLT relations and the single-valued projection on the $\alpha^{\prime}$-expansion. An important open problem is to generalize these relations to genus one, at least for the open and closed partial amplitude building blocks (4.3) and (5.4). 
For the KLT relations, one possible approach is the one presented at tree-level in section 2, based on viewing closed string building blocks as special values of conformal correlation functions, which have a holomorphic factorisation on conformal blocks also at higher genus [39, 40]. At tree-level, this conformal block factorisation gives back the KLT relations, whence come the hope for generalized KLT relations, in particular thanks to the chiral splitting of the closed string integrand on a product of open string integrands at fixed loop momenta [41]. Another possible approach is based on generalizing the interpretation of the KLT relations in terms of twisted de Rham cohomology [49, 50] to higher-loop order [100-103].

A related open problem is to prove the conjectures which connect genus-one open and closed building blocks via the single-valued period formalism. A simpler version of this problem is to prove the conjecture that the coefficients of the constant term of any modular graph function (or form) are single-valued multiple zeta values. The new approach presented in section 4.2 for the two-point case has the potential to be generalized for any number of points, because it is based on the same techniques used in the $N$-point case at genus zero. One must, however, overcome certain technical difficulties which arise already in the three-point case.

Finally, it would also be interesting at one-loop level to study the moduli space integrals which give the full amplitude building blocks. While some results are known in the closed oriented string case $[84,104,105]$, as far as we can tell nothing is known for the other cases. In particular, it would be interesting to understand whether we should expect to generalize in some way the KLT relations and the single-valued projection to the genus-one moduli space integrals. 


\section{References}

[1] Z. Bern, L. J. Dixon, D. C. Dunbar, M. Perelstein and J. S. Rozowsky, "On the Relationship Between Yang-Mills Theory and Gravity and Its Implication for Ultraviolet Divergences”, Nucl. Phys. B 530 (1998) 401 [hep-th/9802162].

[2] Z. Bern, J. J. M. Carrasco, L. J. Dixon, H. Johansson and R. Roiban, "Manifest Ultraviolet Behavior for the Three-Loop Four-Point Amplitude of $\mathcal{N}=8$ Supergravity", Phys. Rev. D 78 (2008) 105019 [arXiv:0808.4112 [hep-th]].

[3] M. B. Green, J. H. Schwarz and L. Brink, " $\mathcal{N}=4$ Yang-Mills and $\mathcal{N}=8$ Supergravity as Limits of String Theories", Nucl. Phys. B 198 (1982) 474.

[4] M. B. Green, J. G. Russo and P. Vanhove, "Non-Renormalisation Conditions in Type II String Theory and Maximal Supergravity”, JHEP 0702 (2007) 099 [hep-th/0610299].

[5] M. B. Green, J. G. Russo and P. Vanhove, "Ultraviolet Properties of Maximal Supergravity", Phys. Rev. Lett. 98 (2007) 131602 [hep-th/0611273].

[6] M. B. Green, J. G. Russo and P. Vanhove, "Modular Properties of Two-Loop Maximal Supergravity and Connections with String Theory”, JHEP 0807 (2008) 126 [arXiv:0807.0389 [hep-th]].

[7] M. B. Green, J. G. Russo and P. Vanhove, "Automorphic Properties of Low Energy String Amplitudes in Various Dimensions", Phys. Rev. D 81 (2010) 086008 [arXiv:1001.2535 [hepth]].

[8] M. B. Green, J. G. Russo and P. Vanhove, "String Theory Dualities and Supergravity Divergences”, JHEP 1006 (2010) 075 [arXiv:1002.3805 [hep-th]].

[9] P. Tourkine and P. Vanhove, "An $R^{4}$ Non-Renormalisation Theorem in $\mathcal{N}=4$ Supergravity", Class. Quant. Grav. 29 (2012) 115006 [arXiv:1202.3692 [hep-th]].

[10] G. Bossard and A. Kleinschmidt, "Supergravity Divergences, Supersymmetry and Automorphic Forms", JHEP 1508 (2015) 102 [arXiv:1506.00657 [hep-th]].

[11] B. Pioline, " $D^{6} R^{4}$ Amplitudes in Various Dimensions", JHEP 1504 (2015) 057 [arXiv: 1502.03377 [hep-th]].

[12] G. Bossard and A. Kleinschmidt, "Cancellation of Divergences Up to Three Loops in Exceptional Field Theory”, JHEP 1803 (2018) 100 [arXiv:1712.02793 [hep-th]].

[13] B. Pioline, "String Theory Integrands and Supergravity Divergences", JHEP 02 (2019), 148 [arXiv:1810.11343 [hep-th]]. 
[14] L. F. Alday, A. Bissi and E. Perlmutter, "Genus-One String Amplitudes from Conformal Field Theory", JHEP 06 (2019), 010 [arXiv:1809.10670 [hep-th]].

[15] S. M. Chester, M. B. Green, S. S. Pufu, Y. Wang and C. Wen, "Modular Invariance in Superstring Theory from $\mathcal{N}=4$ Super-Yang Mills", [arXiv:1912.13365 [hep-th]].

[16] S. M. Chester and S. S. Pufu, "Far Beyond the Planar Limit in Strongly-Coupled N = 4 Sym", arXiv:2003.08412 [hep-th].

[17] M.B. Green, "Modular Properties Of Superstring Amplitudes And Holography", Talk @ ZoomAmplitude 2020.

[18] M. B. Green, J. H. Schwarz and E. Witten, "Superstring Theory. Vol. 1: Introduction", Cambridge, Uk: Univ. Pr. (1987)

[19] J. Polchinski, "String theory. Vol. 1: An introduction to the bosonic string", Cambridge University Press (2007-12-19)

[20] R. Blumenhagen, D. Lüst and S. Theisen, "Basic concepts of string theory", Springer-Verlag Berlin Heidelberg 2013

[21] J. Polchinski and E. Witten, "Evidence for Heterotic - Type I String Duality”, Nucl. Phys. B 460 (1996) 525 [hep-th/9510169].

[22] I. Antoniadis, C. Bachas, C. Fabre, H. Partouche and T. R. Taylor, "Aspects of Type I - Type II Heterotic Triality in Four-Dimensions”, Nucl. Phys. B 489 (1997) 160 [hep-th/9608012].

[23] C. Angelantonj and A. Sagnotti, "Open Strings", Phys. Rept. 371 (2002) 1 Erratum: [Phys. Rept. 376 (2003) no.6, 407] [hep-th/0204089].

[24] M. B. Green and J. H. Schwarz, "Infinity Cancellations in SO(32) Superstring Theory", Phys. Lett. 151B (1985) 21.

[25] M. B. Green and J. H. Schwarz, "The Hexagon Gauge Anomaly in Type I Superstring Theory", Nucl. Phys. B 255 (1985) 93.

[26] E. G. Gimon and J. Polchinski, "Consistency Conditions for Orientifolds and D Manifolds", Phys. Rev. D 54 (1996) 1667 [hep-th/9601038].

[27] C. P. Burgess and T. R. Morris, "Open and Unoriented Strings a La Polyakov", Nucl. Phys. B 291 (1987) 256.

[28] C. P. Burgess and T. R. Morris, “Open Superstrings a La Polyakov”, Nucl. Phys. B 291 (1987) 285.

[29] H. Kawai, D. C. Lewellen and S. H. H. Tye, "A Relation Between Tree Amplitudes of Closed and Open Strings”, Nucl. Phys. B 269 (1986) 1. 
[30] Z. Bern, J. J. M. Carrasco and H. Johansson, "New Relations for Gauge-Theory Amplitudes", Phys. Rev. D 78 (2008) 085011 [arXiv:0805.3993 [hep-ph]].

[31] Z. Bern, J. J. Carrasco, M. Chiodaroli, H. Johansson and R. Roiban, "The Duality Between Color and Kinematics and Its Applications”, arXiv:1909.01358 [hep-th].

[32] J. Broedel, O. Schlotterer and F. Zerbini, "From Elliptic Multiple Zeta Values to Modular Graph Functions: Open and Closed Strings at One Loop”, JHEP 1901 (2019) 155 [arXiv:1803.00527 [hep-th]].

[33] J. E. Gerken, A. Kleinschmidt and O. Schlotterer, "Heterotic-String Amplitudes at One Loop:? Modular Graph Forms and Relations to Open Strings”, JHEP 01 (2019), 052 [arXiv:1811.02548 [hep-th]].

[34] C. R. Mafra and O. Schlotterer, "All-Order Alpha'-Expansion of One-Loop Open-String Integrals", Phys. Rev. Lett. 124 (2020) no.10, 101603 [arXiv:1908.09848 [hep-th]].

[35] C. R. Mafra and O. Schlotterer, "One-Loop Open-String Integrals from Differential Equations: All-Order $\alpha$ '-expansions at $n$ Points”, JHEP 2003 (2020) 007 [arXiv:1908.10830 [hep-th]].

[36] D. Zagier and F. Zerbini, "Genus-Zero and Genus-One String Amplitudes and Special Multiple Zeta Values”, Commun. Num. Theor. Phys. 14 (2020) no.2, 413 [arXiv:1906.12339 [math.NT]].

[37] J. E. Gerken, A. Kleinschmidt and O. Schlotterer, "Generating Series of All Modular Graph Forms from Iterated Eisenstein Integrals”, arXiv:2004.05156 [hep-th].

[38] P. Vanhove and F. Zerbini, "Closed String Amplitudes from Single-Valued Correlation Functions", arXiv:1812.03018 [hep-th].

[39] P. Di Francesco, P. Mathieu and D. Senechal, "Conformal Field Theory", Graduate Texts in Contemporary Physics, New York: Springer-Verlag, 1997.

[40] E. D'Hoker and D. H. Phong, “Conformal Scalar Fields and Chiral Splitting on Superriemann Surfaces”, Commun. Math. Phys. 125 (1989), 469

[41] C. R. Mafra and O. Schlotterer, "Towards the N-Point One-Loop Superstring Amplitude. Part Iii. One-Loop Correlators and Their Double-Copy Structure”, JHEP 08 (2019), 092 [arXiv:1812.10971 [hep-th]].

[42] I. R. Klebanov and L. Thorlacius, “The Size of P-Branes", Phys. Lett. B 371 (1996), 51-56 [arXiv:hep-th/9510200 [hep-th]].

[43] A. Hashimoto and I. R. Klebanov, "Decay of Excited D-Branes", Phys. Lett. B 381 (1996), 437-445 doi:10.1016/0370-2693(96)00621-1 [arXiv:hep-th/9604065 [hep-th]].

[44] M. R. Garousi and R. C. Myers, "Superstring Scattering from D-Branes", Nucl. Phys. B 475 (1996), 193-224 [arXiv:hep-th/9603194 [hep-th]]. 
[45] M. R. Garousi, “Superstring Scattering from O-Planes”, Nucl. Phys. B 765 (2007), 166-184 [arXiv:hep-th/0611173 [hep-th]].

[46] S. Stieberger, “Open \& Closed vs. Pure Open String Disk Amplitudes”, arXiv:0907.2211 [hepth].

[47] Y. X. Chen, Y. J. Du and Q. Ma, "Relations Between Closed String Amplitudes at Higher-Order Tree Level and Open String Amplitudes”, Nucl. Phys. B 824 (2010), 314-330 [arXiv:0901.1163 [hep-th]].

[48] A. Aldi and M. Firrotta, "Closed Superstring Moduli Tree-Level Two-Point Scattering Amplitudes in Type IIB Orientifold on $T^{6} /\left(Z_{2} \times Z_{2}\right)$ ”, arXiv:2001.09468 [hep-th].

[49] S. Mizera, "Combinatorics and Topology of Kawai-Lewellen-Tye Relations", JHEP 1708 (2017) 097 [arXiv: 1706.08527 [hep-th]].

[50] F. Brown and C. Dupont, "Single-valued integration and superstring amplitudes in genus zero", arXiv:1910.01107 [math.NT].

[51] F. Brown, "Single-valued motivic periods and multiple zeta values", Forum Math. Sigma, 2, 2014, arXiv:1309.5309 [math.NT]

[52] F. Brown and C. Dupont, "Single-valued integration and double copy", arXiv:1810.07682 [math.NT]

[53] S. Stieberger, "Constraints on Tree-Level Higher Order Gravitational Couplings in Superstring Theory”, Phys. Rev. Lett. 106 (2011), 111601 [arXiv:0910.0180 [hep-th]].

[54] S. Stieberger, "Closed superstring amplitudes, single-valued multiple zeta values and the Deligne associator", J. Phys. A 47 (2014), 155401 [arXiv:1310.3259 [hep-th]].

[55] S. Stieberger and T. R. Taylor, "Closed String Amplitudes as Single-Valued Open String Amplitudes”, Nucl. Phys. B 881 (2014) 269 [arXiv:1401.1218 [hep-th]].

[56] W. Fan, A. Fotopoulos, S. Stieberger and T. R. Taylor, "SV-map between Type I and Heterotic Sigma Models", Nucl. Phys. B 930 (2018), 195-218 [arXiv:1711.05821 [hep-th]].

[57] O. Schlotterer and O. Schnetz, "Closed Strings as Single-Valued Open Strings: a Genus-Zero Derivation”, J. Phys. A 52 (2019) no.4, 045401 [arXiv:1808.00713 [hep-th]].

[58] O. Schnetz, "Graphical functions and single-valued multiple polylogarithms", Commun. Num. Theor. Phys. 08 (2014) 589 [arXiv:1302.6445 [math.NT]].

[59] V. Del Duca, S. Druc, J. Drummond, C. Duhr, F. Dulat, R. Marzucca, G. Papathanasiou and B. Verbeek, "Multi-Regge kinematics and the moduli space of Riemann spheres with marked points", JHEP 1608 (2016) 152 [arXiv:1606.08807 [hep-th]]. 
[60] E. D’Hoker, M. B. Green, Ö. Gürdogan and P. Vanhove, “Modular Graph Functions”, Commun. Num. Theor. Phys. 11 (2017) 165 [arXiv:1512.06779 [hep-th]].

[61] E. D'Hoker, M. B. Green and B. Pioline, "Asymptotics of the $D^{8} \mathcal{R}^{4}$ Genus-Two String Invariant", Commun. Num. Theor. Phys. 13 (2019), 351-462 [arXiv:1806.02691 [hep-th]].

[62] E. D'Hoker and M.B. Green, “Identities between modular graph forms”, J. Number Theor. 189 (2018), 25-88, [arXiv:1603.00839 [hep-th]].

[63] E. D'Hoker and M. B. Green, “Absence of Irreducible Multiple Zeta-Values in Melon Modular Graph Functions", Commun. Num. Theor. Phys. 14 (2020) no.2, 315 [arXiv:1904.06603 [hepth]].

[64] J. Broedel, C. R. Mafra, N. Matthes and O. Schlotterer, "Elliptic multiple zeta values and one-loop superstring amplitudes”, JHEP 1507 (2015) 112 [arXiv:1412.5535 [hep-th]].

[65] J. Broedel, N. Matthes and O. Schlotterer, "Relations Between Elliptic Multiple Zeta Values and a Special Derivation Algebra”, J. Phys. A 49 (2016) no.15, 155203 [arXiv:1507.02254 [hep-th]].

[66] J. Broedel, N. Matthes, G. Richter and O. Schlotterer, "Twisted elliptic multiple zeta values and non-planar one-loop open-string amplitudes”, J. Phys. A 51 (2018) no.28, 285401 [arXiv:1704.03449 [hep-th]].

[67] E. D’Hoker and D. H. Phong, “The Geometry of String Perturbation Theory”, Rev. Mod. Phys. 60 (1988) 917.

[68] J. Polchinski, "String theory. Vol. 2: Superstring theory and beyond", Cambridge University Press (2007-12-19)

[69] K. Aomoto and M. Kita, "Theory of Hypergeometric Functions." Springer Monographs in Mathematics. Springer Japan, (2011).

[70] V.A. Vasil'ev, I.M. Gel'fand, A.V. Zelevinskii, “General hypergeometric functions on complex Grassmannians”, Funct. Anal. Prilozhen. 21, 19-31 (1987)

[71] I.M Gel'fand, “General theory of hypergeometric functions”, Soviet Math. Dokl. 33 (1986), 573-577.

[72] S. Mizera, "Inverse of the String Theory KLT Kernel”, JHEP 1706 (2017) 084 [arXiv: 1610.04230 [hep-th]].

[73] N. E. J. Bjerrum-Bohr, P. H. Damgaard and P. Vanhove, "Minimal Basis for Gauge Theory Amplitudes", Phys. Rev. Lett. 103 (2009) 161602 [arXiv:0907.1425 [hep-th]].

[74] N. E. J. Bjerrum-Bohr, P. H. Damgaard, T. Sondergaard and P. Vanhove, "The Momentum Kernel of Gauge and Gravity Theories", JHEP 1101 (2011) 001 [arXiv:1010.3933 [hep-th]]. 
[75] C. R. Mafra, O. Schlotterer and S. Stieberger, "Complete N-Point Superstring Disk Amplitude I. Pure Spinor Computation”, Nucl. Phys. B 873 (2013) 419 [arXiv:1106.2645 [hep-th]].

[76] J. Broedel, O. Schlotterer, S. Stieberger and T. Terasoma, “All order $\alpha^{\prime}$-expansion of superstring trees from the Drinfeld associator", Phys. Rev. D 89 (2014) no.6, 066014 [arXiv:1304.7304 [hep-th]].

[77] F. Brown. Single-valued multiple polylogarithms in one variable. C.R. Acad. Sci. Paris, 338:527532, 2004.

[78] F. Brown, "Single-valued hyperlogarithms and unipotent differential equations." preprint on webpage at www . ihes. fr/ brown/RHpaper5.pdf, 2004.

[79] E. Witten, "Superstring Perturbation Theory Revisited", [arXiv:1209.5461 [hep-th]].

[80] R. Donagi and E. Witten, "Supermoduli Space is Not Projected”, Proc. Symp. Pure Math. 90 (2015) 19 [arXiv:1304.7798 [hep-th]].

[81] A. Sen, "Off-Shell Amplitudes in Superstring Theory", Fortsch. Phys. 63 (2015) 149 [arXiv:1408.0571 [hep-th]].

[82] A. Sen and E. Witten, "Filling the Gaps with Pco's", JHEP 1509 (2015) 004 [arXiv: 1504.00609 [hep-th]].

[83] Z. Bern and D. A. Kosower, "A New Approach to One Loop Calculations in Gauge Theories", Phys. Rev. D 38 (1988) 1888.

[84] M. B. Green, J. G. Russo and P. Vanhove, "Low Energy Expansion of the Four-Particle Genus-One Amplitude in Type II Superstring Theory”, JHEP 0802 (2008) 020 [arXiv:0801.0322 [hep-th]].

[85] F. Zerbini, "Elliptic multiple zeta values, modular graph functions and genus 1 superstring scattering amplitudes”, Diss., Univ. Bonn 2017, Bonn, 2018. [arXiv:1804.07989 [math-ph]].

[86] E. Panzer, Talk at the conference "Elliptic integrals in mathematics and physics 2018", held at ETH Zürich-Ascona.

[87] F. Zerbini, "Single-valued multiple zeta values in genus 1 superstring amplitudes", Commun. Num. Theor. Phys. 10 (2016) 703 [arXiv:1512.05689 [hep-th]].

[88] E. D'Hoker, M.B. Green, and P. Vanhove, “On the modular structure of the genus-one type II superstring low energy expansion”, JHEP 08 (2015), 041, [arXiv:1502.06698 [hep-th]].

[89] E. D'Hoker and J. Kaidi, "Hierarchy of Modular Graph Identities", JHEP 11 (2016), 051 [arXiv:1608.04393 [hep-th]]. 
[90] J. E. Gerken, A. Kleinschmidt and O. Schlotterer, "All-order differential equations for one-loop closed-string integrals and modular graph forms”, JHEP 01 (2020), 064, [arXiv:1911.03476 [hep-th]].

[91] J. E. Gerken, "Basis Decompositions and a Mathematica Package for Modular Graph Forms", [arXiv:2007.05476 [hep-th]].

[92] E. D’Hoker and W. Duke, "Fourier Series of Modular Graph Functions", arXiv:1708.07998 [math.NT].

[93] C. R. Mafra and O. Schlotterer, "The Structure of N-Point One-Loop Open Superstring Amplitudes", JHEP 1408 (2014) 099 [arXiv:1203.6215 [hep-th]].

[94] M. B. Green, C. R. Mafra and O. Schlotterer, "Multiparticle One-Loop Amplitudes and S-Duality in Closed Superstring Theory”, JHEP 1310 (2013) 188 [arXiv:1307.3534 [hep-th]].

[95] L. Cappiello, R. Marotta, R. Pettorino and F. Pezzella, "Off-Shell Amplitudes for Nonoriented Closed Strings”, Mod. Phys. Lett. A 13 (1998) 2845 [hep-th/9808164].

[96] B. Enriquez. Analogues elliptiques des nombres multizétas. Bull. Soc. Math. France, 144(3):395_427, 2016, [arXiv:1301.3042 [math.NT]]

[97] F. Brown, "A class of non-holomorphic modular forms I", Res. Math. Sci., 5, 2018, [arXiv:1707.01230 [math.NT]].

[98] J. E. Gerken, A. Kleinschmidt and O. Schlotterer, "Generating series of all modular graph forms from iterated Eisenstein integrals", [arXiv:2004.05156 [hep-th]].

[99] F. Zerbini, "Modular and Holomorphic Graph Functions from Superstring Amplitudes", Conference Proceedings Elliptic Integrals, Elliptic Functions and Modular Forms in Quantum Field Theory, Chap. 18, 459-484 (Springer-Verlag Wien), [arXiv:1807.04506 [math-ph]]

[100] P. Tourkine and P. Vanhove, "Higher-Loop Amplitude Monodromy Relations in String and Gauge Theory”, Phys. Rev. Lett. 117 (2016) no.21, 211601 [arXiv:1608.01665 [hep-th]].

[101] S. Hohenegger and S. Stieberger, "Monodromy Relations in Higher-Loop String Amplitudes", Nucl. Phys. B 925 (2017) 63 [arXiv:1702.04963 [hep-th]].

[102] E. Casali, S. Mizera and P. Tourkine, "Monodromy Relations from Twisted Homology”, JHEP 1912 (2019) 087 [arXiv:1910.08514 [hep-th]].

[103] E. Casali, S. Mizera and P. Tourkine, "Loop Amplitudes Monodromy Relations and ColorKinematics Duality”, [arXiv:2005.05329 [hep-th]].

[104] E. D’Hoker, “Integral of two-loop modular graph functions”, JHEP 06 (2019), 092 [arXiv:1905.06217 [hep-th]]. 
[105] E. D'Hoker and M. B. Green, "Exploring transcendentality in superstring amplitudes", JHEP 07 (2019), 149 [arXiv:1906.01652 [hep-th]]. 